\title{
Michel BERGÈS
}

Professeur des universités, Agrégé de science politique

Université de Bordeaux IV Montesquieu

(2008)

\section{“Claude Lévi-Strauss et les réseaux : parenté et politique.”}

Un document produit en version numérique par Jean-Marie Tremblay, bénévole, professeur de sociologie au Cégep de Chicoutimi

Courriel: jean-marie tremblay@uqac.ca

Site web pédagogique : http://www.uqac.ca/jmt-sociologue/

Dans le cadre de: "Les classiques des sciences sociales" Une bibliothèque numérique fondée et dirigée par Jean-Marie Tremblay, professeur de sociologie au Cégep de Chicoutimi Site web: http://classiques.uqac.ca/

Une collection développée en collaboration avec la Bibliothèque Paul-Émile-Boulet de l'Université du Québec à Chicoutimi

Site web: http://bibliotheque.uqac.ca/ 


\section{Politique d'utilisation de la bibliothèque des Classiques}

Toute reproduction et rediffusion de nos fichiers est interdite, même avec la mention de leur provenance, sans l'autorisation formelle, écrite, du fondateur des Classiques des sciences sociales, Jean-Marie Tremblay, sociologue.

Les fichiers des Classiques des sciences sociales ne peuvent sans autorisation formelle:

- être hébergés (en fichier ou page web, en totalité ou en partie) sur un serveur autre que celui des Classiques.

- servir de base de travail à un autre fichier modifié ensuite par tout autre moyen (couleur, police, mise en page, extraits, support, etc...),

Les fichiers (.html, .doc, .pdf., .rtf, .jpg, .gif) disponibles sur le site Les Classiques des sciences sociales sont la propriété des Classiques des sciences sociales, un organisme à but non lucratif composé exclusivement de bénévoles.

Ils sont disponibles pour une utilisation intellectuelle et personnelle et, en aucun cas, commerciale. Toute utilisation à des fins commerciales des fichiers sur ce site est strictement interdite et toute rediffusion est également strictement interdite.

L'accès à notre travail est libre et gratuit à tous les utilisateurs. C'est notre mission.

Jean-Marie Tremblay, sociologue

Fondateur et Président-directeur général, LES CLASSIQUES DES SCIENCES SOCIALES. 
Cette édition électronique a été réalisée par Jean-Marie Tremblay, bénévole, professeur de sociologie au Cégep de Chicoutimi à partir de :

\section{Michel BERGÈS}

“Claude Lévi-Strauss et les réseaux : parenté et politique”.

Un article publié dans la revue électronique KLESIS - Revue philosophique, no 10, 2008, pp. 1-33. Numéro intitulé : "Hommage à Claude Lévi-Strauss.”

[Autorisation formelle accordée par l'auteur le 31 décembre 2008 de diffuser cette œuvre dans Les Classiques des sciences sociales.]

Courriel : michel.berges@free.fr

Polices de caractères utilisée :

Pour le texte: Times New Roman, 12 points.

Pour les citations : Times New Roman, 12 points.

Pour les notes de bas de page : Times New Roman, 12 points.

Édition électronique réalisée avec le traitement de textes Microsoft Word 2008 pour Macintosh.

Mise en page sur papier format : LETTRE (US letter), 8.5'’ x 11'’)

Édition numérique réalisée le $1^{\mathrm{er}}$ janvier 2009, revu et augmenté le 28 janvier 2009 à Chicoutimi, Ville de Saguenay, province de Québec, Canada.

\section{Fait avec}

Macintosh 


\section{Table des matières}

$\underline{\text { Introduction }}$

Première Partie. Réseau et structure sociale: deux approches inversées

I. Radcliffe-Brown : des réseaux à la structure

I. 1. La structure sociale est un réseau

I. 2. Au-delà des réseaux: l'institution et l'organisation

II. Claude Lévi-Strauss : des « systèmes » symboliques aux réseaux

II. 1. La structure sociale est une institution symbolique systémique

II. 2. De la structure aux réseaux sociaux concrets et au concept de «maison»

Seconde Partie. De la parenté au politique

I. La transfiguration chrétienne de la parenté réelle

II. «Quasi-parenté élargie » et construction des formes politiques modernes 


\section{Michel BERGÈS}

Professeur des universités, Agrégé de science politique

Université de Bordeaux IV Montesquieu

“Claude Lévi-Strauss et les réseaux : parenté et politique”.

Un article publié dans la revue électronique KLESIS — Revue philosophique, no 10, 2008, pp. 1-33. Numéro intitulé : "Hommage à Claude Lévi-Strauss.”

\section{Introduction}

$\underline{\text { Retour à la table des matières }}$

Dans un manuel récent d'initiation à l'anthropologie, Claude Rivière met l'accent sur la dimension réticulaire de la parenté, au fondement des sociétés traditionnelles, en ces termes :

« La parenté, du point de vue biologique, relève de la nature, mais elle est encore plus lien juridique et code moral, car la société attribue aux représentations mentales concernant le système et les liens de parenté un pouvoir de contrainte et de normativité. Un système de parenté, ni agrégat structuré, ni groupe social, est un réseau complexe de liens aux nombreuses ramifications $» 1$.

On peut alors se demander comment le mot et l’objet « réseau », développés dans un sens plus ou moins différent par la sociologie ${ }^{2}$, ont été théorisés par l'anthropologie. Ne relèveraient-ils que d'un usage métaphorique « constructiviste » ? À l’inverse, les réseaux de parenté auraient-ils une réalité concrète, liée à des stratégies conscientes de la part de divers groupes sociaux (maisonnées rassemblées en villages, clans, lignages, parentèles, tribus, chefferies etc.) et des individus qui s’y rattachent? Par ailleurs, peut-on articuler une théorie des réseaux concernant les sociétés traditionnelles, fondamentalement orales, avec ce que l'on

1 Claude Rivière, Introduction à l'anthropologie, Paris, Hachette supérieur, « Les fondamentaux », 1995, p. 52.

2 Alain Degenne et Michel Forsé, Les Réseaux sociaux. Une analyse structurale en sociologie, Paris, Armand Colin, col. «U sociologie », 1994. 
observe de réticulaire dans des sociétés plus complexes et plus nombreuses connaissant l'écriture, susceptibles de "conscientiser », en les réglementant par écrit, les institutions réticulaires pratiques? De façon générale, comment les réseaux de parenté s'articulent-ils à des réseaux plus vastes et quelle est la portée comparative d'un tel élargissement?

Pour tenter de répondre, il apparaît incontournable d'interroger en premier lieu l'anthropologie sociale. Malgré l'évidence de l'usage du mot « réseau » dans cette discipline, un constat s'impose : à l'instar d'un des premiers manuels publiés en France par Marcel Mauss en $1947^{3}$, celui-ci est peu présent dans les index, traités, dictionnaires - même les plus récents ${ }^{4}$. Les « réseaux » se retrouvent cependant conjugués plus ou moins explicitement par les paradigmes de l'ethnologie sociale, culturelle, symbolique, structurale-systémique ou dynamique ${ }^{5}$, sur le plan théorique de la définition de la « structure sociale ».

Dans un premier temps, nous verrons comment, à travers deux interprétations classiques, celle-ci est de fait associée au concept de réseau : d'un côté, celle d'Alfred Reginald Radcliffe-Brown, tenant d'une anthropologie "concrète » et « naturaliste », étayée par une méthode « aristotélicienne » (I) ; de l'autre, celle de Claude Lévi-Strauss, défenseur d'une anthropologie structuraliste " formelle » et symbolique, qu'il qualifie de « galiléenne » (II). Qu’en est-il précisément pour cet

3 Marcel Mauss, Manuel d'ethnographie, Paris, Payot, 1971.

4 C'est le cas du moins des ouvrages classiques d'initiation: J. A. Mauduit, Manuel d'ethnographie, Paris, Payot, 1960 ; Robert Lowie, Traité de sociologie primitive, Paris, Payot, 1969 ; E. E. Evans-Pritchard, Anthropologie sociale, Paris, Payot, 1969 ; Georges Balandier, Anthropologie politique, Paris, PUF, 1969 ; Marcel Mauss, Manuel d'ethnographie, op. cit. ; Michel Panoff et Michel Perrin, Dictionnaire de l'ethnologie, Paris, Payot, 1973 ; Norbert Rouland, Anthropologie juridique, Paris, PUF, « Droit fondamental », 1988 ; Christian Ghasarian, Introduction à l'étude de la parenté, Paris, Éditions du Seuil, 1996 ; Robert Deliège, Anthropologie de la parenté, Paris, Armand Colin, col. «Cursus », 1996 ; Marc Abelès et Henri-Pierre Jeudy, Anthropologie du politique, Paris, Armand Colin, col. « U », 1997 ; Claude Rivière, Introduction à l'anthropologie, op. cit., et Anthropologie politique, Paris, Armand Colin, col. "Cursus », 2000 ; Abécédaires de Claude Lévi-Strauss, Paris, Vrin, 2008.

5 Nous empruntons cette différenciation des courants théoriques de l'anthropologie à François Laplantine, L'Anthropologie, Paris, Payot, 1995. 
objet « moins beau » peut-être que les mythes, mais au cœur tout de même des structures élémentaires et complexes de la parenté ?

Dans un second temps, nous apprécierons l'apport de tels débats aux diverses disciplines concernées par la problématique de la parenté via son articulation avec les mécanismes d’élargissement des réseaux sociaux en œuvre au niveau des processus de construction du politique dans les sociétés complexes, « à écriture ».

\section{Première Partie Réseau et structure sociale : deux approches inversées}

\section{Radcliffe-Brown : des réseaux à la structure}

$\underline{\text { Retour à la table des matières }}$

Après la période des pères fondateurs (Henry Maine, J. F. Mac Lennan, John Lubbock, James Frazer), c'est Alfred Reginald Radcliffe-Brown, unique étudiant d'un professeur venu de la psychologie à Cambridge (W. H. R. Rivers), qui, dès 1904, orienta les recherches de l'anthropologie sociale anglaise sur les relations de parenté, de mariage et d'affinité, suivant d'ailleurs en cela les travaux pionniers de l’Américain Lewis Henry Morgan ${ }^{6}$.

Dans l'introduction de Structure et fonction dans la société primitive, publié en 1952, Radcliffe-Brown ramassa sa conception «théorique », qu’il voulait non historique, des institutions sociales des sociétés traditionnelles (la théorie n’étant pour lui qu' « un schéma d'interprétation applicable à une classe déterminée de phénomènes en vue de la comprendre », à partir d'observations, de comparaisons, de classifications). Citant explicitement Montesquieu, Comte, Durkheim, Spencer, il se réclama à l'occasion d'une posture « vieille de deux siècles » bâtie autour de

6 Sur l'histoire de l'anthropologie anglaise, cf. Evans-Pritchard, Anthropologie sociale, op. cit. 
«trois concepts fondamentaux»: ceux de "processus », de «structure » et de « fonction », articulés eux-mêmes autour des objets « réseau » et « institution ».

\section{1. La structure sociale est un réseau}

$\underline{\text { Retour à la table des matières }}$

Pour Radcliffe-Brown, le processus de la vie sociale est composé d'actions, de transactions et d'interactions d'êtres humains agissant individuellement ou en groupe selon certaines régularités. Celles-ci constituent un «système » (idée inventée par Montesquieu), en ce sens qu'elles sont solidaires, interdépendantes et en interconnexion, mais aussi «cultivées » et transmises d'une génération à l'autre, donc conscientes et construites par les acteurs qui les portent. Les relations sociales dégagent des formes régulières, répétées, cohérentes, qui ne peuvent être classés en « espèces » (comme les formes de la vie organique), mais plutôt en «types ». Ce sont ces types que l'anthropologie sociale doit rechercher à partir d'une comparaison d'études de cas. Le maintien de certaines formes permet de comprendre l'existence d'un système social, mais aussi les modes d'adaptation des hommes à ce système. Cela à trois niveaux qui assument sa stabilité : l'adaptation écologique à l'environnement physique ; l'adaptation des relations à la coopération nécessaire au maintien du système grâce à des institutions d'ordre, de régulation des conflits et de répression ; l'adaptation mentale par la culture, afin d'inculquer les normes de reproduction du système ainsi que sa transmission.

Pour ce qui est des sociétés " primitives », l'essentiel de la structure sociale repose sur un "système de parenté » qui a comme unité structurante la famille élémentaire (un homme, son épouse, leurs enfants par naissance ou adoption), vivant ou non ensemble sous un toit commun. Trois types de relations en émergent alors, dîtes de « premier ordre » : entre parents et enfants des mêmes parents, entre enfants des mêmes parents, entre mari et femmes comme parents du ou des mêmes enfants. Des relations « de second ordre » ou de n ordre s'établissent entre deux ou $\mathrm{n}$ familles élémentaires ayant un ou des membres communs. L’anthropologue anglais décrit ainsi ces relations : 
«Cet emboîtement de familles élémentaires créé ce que j’appellerai, faute d'un meilleur terme, un réseau de relations généalogiques à extension indéfinie (a network of genealogical relations) ${ }^{7}$.

Malgré des hésitations, donc, l'aspect réticulaire des relations sociales observables sur le terrain est souligné en ces termes :

« Il est rare de trouver une communauté absolument isolée, sans contact extérieur. De nos jours, le réseau des relations sociales s'étend sur le monde entier, sans aucune solution de continuité, nulle part. Dans une région bien choisie et de taille convenable, on peut étudier le système structural qui y apparaît, c'est-à-dire le réseau de relations liant les habitants aussi bien entre eux qu'avec les peuples des autres régions ${ }^{8}$.

La difficulté réside dans le fait que le lien de parenté dépasse la famille domestique fondatrice (qui reste bilatérale), la maisonnée, les regroupements de foyers de proches, notamment dans le clan (gens, qui, lui, est toujours unilatéral en ce sens qu'il institue une parenté soit avec le père, soit avec la mère, mais jamais avec les deux), distinct de la lignée et plus large qu'elle.

Mais quelle que soit son extension, la parenté simple ou élargie, bilatérale ou unilatérale, implique un « statut » des individus. Celui-ci repose sur un ensemble de droits, c'est-à-dire de contraintes, de tabous et de devoirs concernant les rapports, soit d'une personne à une personne, soit d'une personne à un ensemble de personnes, soit de diverses personnes à une chose. Ces relations statutaires sont programmées par une « structure sociale » juridique, que Radcliffe-Brown assimile à « un réseau total », construit et conscient :

«Aussi le système de parenté est-il un réseau de relations sociales d'un type exactement défini et constitue-t-il par là une partie de ce réseau total des relations sociales que j'appelle structure sociale. Font

7 A. R. Radcliffe-Brown, Structure et fonction dans la société primitive, Paris, Éditions de Minuit, « Points Sciences humaines », 1972, p. 116.

8 Ibid., p. 278. 
partie du système, les droits et devoirs réciproques des parents et les coutumes sociales observées dans leurs contacts sociaux, qui permettent la description des relations sociales. Le culte des ancêtres, là où il existe, est une partie réellement significative du système de parenté, puisqu'il est constitué par les relations liant les personnes vivantes à leurs parents défunts et qu'il affecte les relations réciproques de ces personnes vivantes. Les termes qu'une société emploie pour désigner les parents font partie du système ; tout comme les idées que les gens eux-mêmes se font de leur parenté ${ }^{9}$.

Ce « réseau généalogique » s’insère dans une unité complexe, représentée et organisée qui oriente les relations de façon symétrique ou asymétrique au niveau des interdépendances, des associations, des alliances, voire des appartenances à une société politique. Dissemblables par rapport à des relations contractuelles consenties, celles-ci peuvent être décrites concrètement et comparées d'une société à l'autre, même si elles sont chaque fois spécifiques. On doit se garder de les enfermer dans des «abstractions généralisantes », dans un système universel caractérisant mécaniquement un mode unique de structuration. Ces relations réticulaires sont localisées dans l'espace et dans le temps.

La structure sociale ne se limite cependant pas aux relations sociales apparentes qui, d'ailleurs, n’impliquent pas toujours une dimension structurée. Par contre, en tant que telle, elle se manifeste de façon réticulaire. Radcliffe Brown parle là du « réseau de relations sociales existant réellement »10, dont la reconstitution reste l'objectif scientifique principal de l'anthropologie sociale » :

« L'étude des structures sociales ne se ramène donc pas exactement à celle des relations sociales, malgré la définition que donnent certains sociologues. Une relation particulière entre deux personnes, à moins qu'il ne s'agisse d'Adam et Ève dans le Paradis terrestre, n'est qu'une partie d'un réseau plus vaste de relations sociales liant de nombreuses autres personnes : ce réseau constitue notre objet de recherche » 11 .

$9 \quad$ Ibid., p. 118-119.

10 Ibid., p. 274.

11 Ibid., p. 275. 
L'anthropologue précise qu'il se démarque d'Evans-Pritchard qui en reste, lui, à la description de relations concrètes établies par des groupes sociaux permanents (les villages, les clans, les tribus, les nations) conservant leur identité et leur continuité au-delà de tout changement. Il faut plutôt élargir et inverser l'objet et le concept de réseau, puisqu'il est à la fois relation et structure. Le réseau relationnel exprime le social mais ne le condense pas totalement. Le lien social dépend d'une structure réticulaire plus globale à découvrir. Celle-ci comprend, certes, l'ensemble des relations concrètes entre les agents (par exemple, dans une tribu, le " réseau de relations semblables de personne à personne »), mais aussi le rôle social des personnes, qui sépare individus et groupes (établi " grâce aux liens généalogiques »), de même que les vivants et les morts. La " personnalité sociale » marque la position d'un individu dans cette structure plus vaste que ses échanges quotidiens, position dont il est nécessairement conscient puisqu'il reproduit sans cesse et transmet à ses descendants ladite structure. Les relations sociales n’apparaissent donc pas épuisables dans la réalité historique ou géographique, de leur déploiement. Elles relèvent de cet agencement généalogique qui constitue une « forme structurale » comparable, d'une unité territoriale à l'autre, alors même que ces unités se différencient entre elles au niveau de leurs réseaux relationnels. Les relations concrètes, qui ne sont pas la forme structurale, peuvent changer, mais point cette dernière ${ }^{12}$. La structure est en fait un réseau persistant, réel, qui dépasse ses membres :

« Les phénomènes sociaux observables dans toute société humaine ne résultent pas immédiatement des individus humains qui la composent, mais résultent de la structure sociale qui les unit ${ }^{13}$.

Mais d'où vient alors le caractère unificateur de ladite structure ? Serait-on en présence d'une définition tautologique ? Quelle relation établir entre structure et réseau, si la structure dépasse le réseau des relations sociales, même si elle revêt une forme réticulaire ? Serait-elle une entité au-dessus des réseaux concrets ?

12 Ibid., p. 278.

13 Ibid., p. 275. 


\section{2. Au-delà des réseaux : l'institution et l'organisation}

Retour à la table des matières

Réponse de Radcliffe-Brown: l'aspect structural découle de l'existence d' « institutions », objet essentiel qui se résume dans les « positions sociales différentielles " des individus. L'anthropologie, attachée à découvrir l'ordonnancement d'une société particulière, doit alors étudier dans sa partie théorique (suivant en cela, en l'adaptant, l'exemple de la science biologique) trois objets complémentaires et inséparables. D’abord, de façon «morphologique », des "systèmes structuraux » (ou " structure sociale ») déterminant des réseaux de relations comparables à partir d'institutions sociales. Ensuite, dans une approche " physiologique », des "fonctions sociales » (morale, loi, religion, gouvernement, éducation...), qui assument la pérennité de la structure, conserve le « réseau des relations sociales » et son fonctionnement à l'identique. Enfin, des «processus » de changement qui font surgir de nouvelles formes structurales ${ }^{14}$.

Pour révéler cet ordre social, il faut dépasser les approches qui théorisent une structure abstraite, ainsi que les analyses de cas qui historicisent à outrance les relations sociales et les enferment dans des espaces irréductibles non comparables. La structure d'un type de société ne peut être comprise qu'a posteriori, à partir de la comparaison de nombreux cas dont les aspects visibles apparaissent dissemblables, mais dont la forme reste identique. Or, celle-ci est produite par des normes intériorisées. En effet, toutes les relations directes et indirectes par rapport à la structure réticulaire d'une société donnée sont déterminées par des modèles de comportements (tabous, étiquette, morale, droit, règlement des conflits, logiques d'intérêts, valeurs...), auxquels se conforment les individus et les groupes. Ces modèles de programmation des réseaux concrets de relations sociales, ce sont précisément les « institutions » :

« Les institutions sociales comprises comme des modes normalisés de comportement, constituent le mécanisme qui conditionne l'existence et

14 Ibid., p. 281. 
la permanence d'une structure sociale ou d'un réseau de relations sociales » 15 .

C’est grâce à ces «institutions » et à leur intériorisation par les groupes que les réseaux sociaux sont durables, mais aussi grâce à leur caractère "systémique ». Leurs fonctions se complètent, en « une disposition ordonnée de parties ou d'éléments composant un tout qui agit avec continuité » :

«Les relations sociales dont le réseau continu constitue la structure sociale, ne sont pas des conjonctions fortuites d'individus, mais sont déterminées par le processus social. Toute relation de ce genre implique que la conduite des personnes dans leurs interactions réciproques obéisse à des règles ou à des modèles » 16 .

Ces «programmes », ces codes institutionnels impliquent que chacun attend qu'autrui se comporte d'une façon identique par rapport à la sienne, à travers des normes de conduites établies, reconnues et réciproques. Les modèles institutionnels établissent donc une double relation au système social. D'abord, ils fournissent des normes-repères aux réseaux. Ensuite, ils sont intériorisés par les groupes qui reconnaissent et pratiquent les normes en question. Les institutions ont pour fonction d'établir la relation entre la structure sociale et le processus de vie des relations ordinaires.

Les réseaux sociaux relèvent aussi d'une " organisation » (distincte d'une « institution ») qui ordonne les activités considérées de façon régulière et spécifique d'un même type de société à une autre, mais aussi selon les circonstances traversées. L'institution implique une permanence. L'organisation, toujours concrète et événementielle, révèle, elle, une adaptation temporelle et spatiale.

Ainsi, l'anthropologie anglaise de Radcliffe-Brown définit initialement la structure sociale à partir des réseaux pour les dépasser en les enchaînant à l'institution et à l'organisation. On sait que Claude Lévi-Strauss, foncièrement opposé à une telle définition, a déclaré, au sujet de son vis-à-vis britannique, « ob-

15 Ibid., p. 287.

16 Ibid., p. 67. 
servateur, analyste et classificateur incomparable », qu’il décevait souvent lorsqu'il se voulait théoricien ${ }^{17}$. Ces remarques « de bonne guerre » augurent une inversion de problématique. En effet, le structuralisme définit la structure sociale en tant qu'institution symbolique primordiale. Cependant il retrouve a posteriori l’objet réticulaire concret ainsi que le concept de réseau qu'il semblait écarter à l’origine...

\section{Claude Lévi-Strauss : des « systèmes » symboliques aux réseaux}

$\underline{\text { Retour à la table des matières }}$

L’ethnologue français a précisé sa définition de la structure sociale en 1952, puis en 1958 18. Celle-ci revêt apparemment deux aspects contradictoires 19 .

D’un côté, Les Structures élémentaires de la parenté ou Anthropologie structurale I et II, relèvent d'un constructivisme objectiviste qui refuse de réduire le social à un objet immédiatement saisissable et concrètement « relationnel ».

De l'autre, l'enseignement au Collège de France sur la pluralité des systèmes de parenté, leur évolution, leur confrontation à l’histoire (résumé de façon lumineuse dans Paroles données ${ }^{20}$ ), révèle une ouverture insoupçonnée qui atténue

17 Lévi-Strauss, Anthropologie structurale, Paris, Plon, Presse Pocket, 1958, p. 336.

18 Anthropologie structurale, op. cit., p. 329-378, et Unesco, Sens et usages du mot structure, La Haye, Paris, Mouton, 1972, p. 40-45, 143-145, 150, 155, 157.

19 L'opposition théorique entre Claude Lévi-Strauss et divers anthropologues anglais a été résumée par Louis Marin dans sa présentation de l'édition française de Structure et fonction dans la société primitive. Cf. Radcliffe Brown, Structure et fonction dans la société primitive, op. cit., p. 5-54. On peut également consulter l'ouvrage collectif, Sens et usages du terme structure, publié à partir d'un colloque sous l'égide de l'Unesco, désireuse de constituer un dictionnaire terminologique des sciences sociales, qui s'est tenu à Paris du 10 au 12 janvier 1959. L’ouvrage de Jean Viet, Les Méthodes structuralistes dans les sciences sociales, La Haye, Paris, Mouton, 1967, donne un excellent résumé des débats à ce propos.

20 Lévi-Strauss, Paroles données, Paris, Plon, 1984. 
les oppositions avec Radcliffe-Brown et réintroduit l’objet et le concept de « réseau concret ». Existerait-il là « deux » Claude Lévi-Strauss ?

\section{1. La structure sociale est une institution symbolique systémique}

$\underline{\text { Retour à la table des matières }}$

Le « premier Lévi-Strauss », explorateur de « l’atome de parenté » ${ }^{21}$ comme de la règle universelle de la prohibition de l'inceste, se devait de se démarquer des fondateurs anglais de l'anthropologie sociale, ne serait-ce que pour réaliser son idée d'une science sociale universaliste au-dessus des scories de l'historicisme, du culturalisme, ou de la théorie fonctionnaliste «naturaliste » et «biologiste », en perpétuant ainsi la tradition sociologique française (plus proche de Marcel Mauss que d'Émile Durkheim cependant ${ }^{22}$ ).

S’il reconnaît à Radcliffe-Brown d’indéniables « qualités d’observateur », des « titres de gloire » dans l'histoire de la pensée structurale, ainsi qu’une « mémorable réussite déductive », Claude Lévi-Strauss écarte sa définition réticulaire de la structure sociale. Il préfère privilégier les travaux de Morgan (à la mémoire duquel il a dédié sa thèse, Les Structures élémentaires de la parenté). Si Spencer utilise le mot « structure », en ignorant la chose, Morgan écarte le mot, mais étudie la chose dans ses travaux sur les Iroquois ${ }^{23}$. Le structuralisme n’a pas été transmis par les pères fondateurs (Taylor, Morgan, Spencer...), mais fut plutôt « redécouvert » ultérieurement, grâce à Marcel Mauss et à d’autres influences (le marxisme, la géologie et la psychanalyse) ${ }^{24}$. Radcliffe-Brown, lui, préférant Spencer, a confondu, dans son « empirisme réaliste », relations sociales et structure sociale. D’où ce jugement sans appel :

21 C'est-à-dire du système quadrangulaire de relations entre frère et sœur, mari et femme, père et fils, oncle maternel et neveu, cf. Lévi-Strauss, Anthropologie structurale II, Paris, Plon, 1973, p. 105.

22 Lévi-Strauss, Anthropologie structurale II, op. cit., «Ce que l'ethnographie doit à Durkheim », p. 57-62.

23 Unesco, Sens et usages du mot structure, op. cit., p. 143.

24 Lévi-Strauss, Tristes tropiques, Paris, Plon, 1955, p. 50. 
« En fait, toute son œuvre réduit la structure sociale à l'ensemble des relations sociales existantes dans une société donnée. Sans doute a-t-il parfois esquissé une distinction entre structure et forme structurale. Mais le rôle qu'il accorde à cette dernière notion est purement diachronique » 25 .

Claude Lévi-Strauss passe vite sur le concept d'institution de son vis-à-vis britannique, pour mettre en avant sa définition effectivement diachronique d'organisation. Mais pour ce dernier, la «structure » empirique constitue une partie de la réalité 26 , « un réseau de relations dyadiques » unissant une personne à une autre. Cela est inconcevable pour l'ethnologue structuraliste :

« Ces relations dyadiques constituent-elles vraiment la matière première de la structure sociale ? Ne sont-elles pas plutôt le résidu - obtenu par analyse idéale - d'une structure préexistante, dont la nature est plus complexe ? $»^{27}$.

On ne peut réduire la structure sociale à une chaîne réticulaire et continue, par addition de conglomérats individuels relationnels, sans cesse allongée d'interactions nouvelles, ce qui la rendrait « extensible indéfiniment ».

À l'inverse, c'est la « structure » qui prévaut sur les relations ! Elle reste inséparable de son contenu, forme des «systèmes » codifiés (parenté, rituels, mythes, cérémonials, façons de cuisiner...). Une société est constituée de ces plans structurés qui n'épuisent pas la connaissance que l'on peut en avoir : certains niveaux se montrent «rebelles à l'analyse structurale », soit en raison d'un manque d'information les concernant, soit à cause de leur «nature irréductible ». Cela d'autant que la réalité sociale reste «mouvante et imprévisible » ${ }^{28}$. Une « structure » implique des propriétés finies, combinées et transformables, donc prévisibles en termes d'expérimentation «algébrique ». Cette caractéristique permet de passer d'un système à un autre et de comprendre leurs rapports. La «modélisa-

25 Lévi-Strauss, Anthropologie structurale, op. cit., p. 361.

26 Lévi-Strauss, Anthropologie structurale II, op. cit., p. 99.

27 Ibid., p. 362.

28 Unesco, Sens et usages du mot structure, op. cit., p. 159. 
tion » de ceux-ci peut être classée dans des groupes de modèles comparables, qu'une démarche scientifique peut concevoir ${ }^{29}$. L'existence de ces « systèmes » symboliques dissimulés par un inconscient social reste une hypothèse théorique, « falsifiable ». Claude Lévi-Strauss affirme sur ce point :

« Plus nette est la structure apparente, plus difficile devient-il de saisir la structure profonde, à cause des modèles conscients et déformés qui s'interposent comme des obstacles entre l'observateur et son objet $» 30$.

Plus tard, il justifiera de façon identique sa conception constructiviste objectiviste :

« La preuve définitive que la matière a une structure moléculaire est dans le microscope électronique, dont l'oculaire donne à voir des molécules réelles; mais les molécules n’en deviendront pas visibles à l'œil nu pour autant. On ne peut davantage attendre de l'analyse structurale qu'elle change la façon dont nous appréhendons les relations sociales telles qu'elles se manifestent concrètement : elle permet seulement de mieux les comprendre. Et si l'on parvient à saisir leur structure, ce ne sera jamais au niveau empirique où elles étaient déjà apparues, mais à un niveau plus profond et resté jusqu'alors inaperçu : celui des catégories inconscientes, qu'on peut espérer atteindre en rapprochant des domaines qui, à première vue, semblaient n'avoir pas de rapports. Ces domaines incluent d'une part les institutions sociales telles qu'elles fonctionnent dans la pratique, et d'autre part, les diverses manières selon lesquelles, dans leurs mythes, leurs rites et leurs représentations religieuses, les hommes essayent de voiler ou de justifier les contradictions entre la société réelle où ils vivent et l’image idéale qu'ils s'en font » ${ }^{31}$.

29 Cf. Jean Piaget (dir.), Logique et connaissance scientifique, Paris, Gallimard, « Encyclopédie de la Pléiade », 1967. Ce dernier parle de « constructivisme » au niveau épistémologique.

30 Lévi-Strauss, Anthropologie structurale, op. cit., p. 335.

31 Lévi-Strauss, Anthropologie structurale II, op. cit., p. 100. 
Autre limite du « réalisme » de Radcliffe-Brown : la mise en avant dans les systèmes de parenté de la cellule biologique de base (la famille au niveau de la relation mari-femme, parents-enfants, frères-sœurs), qui ordonnerait tous les types familiaux. Or, c’est l'inverse que l'on constate. La parenté, fondée sur l'alliance, est culturelle, non biologique. Elle repose sur un système d'échanges de femmes mais aussi de signes, dans une logique de communication sociale structurée comme un langage, que l'on retrouve encore dans l'économie (échange de biens et de services) ou dans la linguistique (échange de messages), comme dans la musique (échange de sons marqués pas des signes qui n’ont pas de sens), dans la cuisine (façon qu'ont les humains d'adapter les produits de la nature pour se nourrir, en les retenant crus, séchés, bouillis, ou cuits), dans les rites (où les vivants s'adressent aux mort), ou dans les mythes (où les morts parlent aux vivants - qui les font parler). Toute culture articule de façon « sauvage », créative à l’infini, ces « jeux communicationnels ». On se trouve en présence de « structures de communication » et de sens plus que de structures de relations interindividuelles ordonnées par des institutions explicites. Autrement dit, pour Claude Lévi-Strauss, l'institution symbolique précède les relations sociales. Le sens prime par rapport à l’interaction. C’est lui qui codifie les échanges communicationnels.

Les liens concrets et oralisés consciemment entre des personnes forment des attitudes de surface, car les agents sont «agis », plus qu' "acteurs ». Ils ne connaissent pas vraiment les codes structurels de sens qui les produisent, même s’ils les désignent plus ou moins adroitement. Les attitudes et les appellations forment un langage, une logique complexe, classificatoire et intelligente, dont il faut recomposer la grammaire. La pensée sauvage pense toujours aussi bien que la pensée domestiquée, « civilisée ». Si l’on réduit tout à des attitudes comportementales extensibles à l'infini, le concept de « structure » disparaît. Pour l'ethnologue français, qui applique à Radcliffe-Brown ses critiques précédentes adressées à l'identique à l'auteur des Formes élémentaires de la vie religieuse, Émile Durkheim, c’est le symbolique qui produit le social, et non l'inverse. Bref, opposition là entre constructivisme idéaliste d'un côté, et relativisme sociologiste objectiviste de l'autre, dans la production des « idées » et des catégories logiques universelles. L’on sait que pour « le second Durkheim» des Formes élémentaires de la Vie religieuse, comme pour l'ensemble de son équipe de L’Année sociologique 
(contre Aristote!), celles-ci étaient relatives et historicisées ${ }^{32}$. Pour le néokantien Claude Lévi-Strauss, anti-durkheimien ici, les catégories mentales restent universelles et fondent toutes les structures de sens des sociétés humaines.

On comprend mieux alors la quasi absence, dans l'ensemble des index construits par l'ethnologue structuraliste et les éditeurs de ses ouvrages, de l'objet « réseau », au sens de Radcliffe-Brown. Le concept de « système » est seul mis en avant pour définir les «structures sociales ». Pour en rester aux métaphores, observons que dans la représentation imagée de celles-ci, sont plutôt valorisés des « diagrammes » arborescents qui décrivent des liaisons verticales, horizontales ou obliques autour de lignes, de «cordes » (mot utilisé par certains indigènes !), d'axes imbriqués (comme les « tuiles d'un toit »), de « lacis d'obligations en torsades le long d'un axe vertical » ${ }^{33}$. L'image analogique (et non homologique) du réseau, qui ne retient pas l'attention de Claude Lévi-Strauss quand il commente les définitions de Radcliffe-Brown, semble faire partie des « illusions inductives » d'une ethnologie «immédiate », accumulant des « informations nombreuses et superficielles » ou « inutilisables ».

Sur le plan épistémologique, l’ethnologue français oppose la « méthode aristotélicienne » de Radcliffe-Brown, faîte de « simples corrélations inductives », à sa "méthode galiléenne », attentive à déterminer plutôt des « lois de variations concomitantes » ${ }^{34}$. Cela implique qu'au lieu de prendre la biologie pour inspiratrice, il faut se tourner vers les sciences qui ont investi l'aspect formel des phénomènes sociaux en introduisant la mesure ou la formalisation, à travers des modèles mécaniques ou statistiques. Le structuralisme lévi-straussien (très proche de l'épistémologie anglo-saxonne, telle que la définit Raymond Aron ${ }^{35}$ ), environné

32 Émile Durkheim, Les Formes élémentaires de la vie religieuse, Paris, Le Livre de Poche, «Classiques de la philosophie », 1991, p. 716-740. [Livre disponible dans Les Classiques des sciences sociales. JMT.]

33 Marcel Leenhardt, quant à lui, préféra utiliser l'image de «petites surfaces elliptiques à deux foyers, s’imbriquant les unes dans les autres, ou celle d'un tissu cellulaire » pour décrire la structure sociale mélanésienne, cf. Marcel Leenhardt, Do Kamo. La personne et le mythe dans le monde mélanésien, $\mathrm{Pa}-$ ris, Gallimard, col. « Tel », 1971, p. 182.

34 Lévi-Strauss, Anthropologie structurale, op. cit., p. 372.

35 Raymond Aron, Leçons sur l'histoire, Paris, Le Livre de Poche, «BiblioEssais », 1989. 
d’une idéologie scientiste liée à la montée en puissance de l'électronique et de la futurologie dans les années cinquante-soixante, recommande alors les ouvrages sur la théorie des jeux et le comportement économique, la cybernétique, la théorie mathématique de la communication ${ }^{36}$ (ainsi est jugée « intéressante » la perspective de Rapoport concernant « le traitement mathématique des phénomènes cycliques de domination chez les poules » ${ }^{37}$ ). Il s'agissait alors de construire une « science nouvelle » attentive aux règles ( «indépendantes des acteurs »), aux jeux, aux parties, aux choix, aux coups, aux stratégies, qui ne se réduisent pas à des comportements conscients (notons ici l'utilisation inversée de concepts behaviouralistes américains !). Bref, au début était l’institution symbolique programmatrice des actes humains ! D’où cette déclaration d'un ethnologue semble-t-il fasciné par « le grand royaume de la communication » 38 :

« La nature des joueurs est indifférente, ce qui compte étant seulement de savoir quand un joueur peut choisir, et quand il ne le peut pas » 39.

Cette indifférence apparente aux valeurs explicites et à la liberté humaine, au profit d'un système logique, formalisable et binaire, actionné et intériorisé mystérieusement par des agents qui semblent ne plus avoir de chair, de nature personnelle, constitua, en son temps, un premier pas constructiviste vers une « mathématique sociale ». Celle-ci devait être fondée sur le croisement de toutes les sciences de la communication ou de la démographie, permettant la conversion réciproque des modèles statistiques et mécaniques. Les systèmes de parenté pouvaient alors s’ouvrir à une formalisation effective, ainsi supputée par le théoricien structuraliste :

36 Lévi-Strauss, Anthropologie structurale, op. cit., p. 337. Claude Lévi-Strauss indique son intérêt pour les ouvrages suivants: Théories des jeux et comportements économiques de J. von Neumann et O. Morgenstern, 1994 ; Cybernétique, de N. Wiener (1948) ; La Théorie mathématique de la communication, de C. Shannon et W. Weaver (1950).

37 Lévi-Strauss, Anthropologie structurale, op. cit., p. 372.

38 Ibid., p. 355-357.

39 Ibid., p. 356. 
«L'“information” d'un système de mariage est fonction du nombre d'alternatives dont dispose l'observateur pour définir le statut matrimonial (c'est-à-dire celui de conjoint possible, prohibé, ou assigné) d'un individu quelconque, par rapport à un prétendant déterminé. Dans un système à moitié exogamiques, cette information est égale à l'unité. Dans une typologie australienne, elle augmente avec le logarithme du nombre des classes matrimoniales. Un système théorique de panmixie (où chacun pourrait épouser n’importe qui) ne présenterait aucune "redondance", puisque chaque choix matrimonial serait indépendant de tous les autres. Inversement, les règles du mariage constituent la redondance du système considéré. On pourra aussi calculer le pourcentage des choix "libres" (non pas absolument, mais par rapport à certaines conditions postulées par hypothèse) qui se produisent dans une population matrimoniale donnée, et assigner une valeur numérique à son “entropie”, relative et absolue » 40 .

Le naturel, le subjectivisme, le réalisme concret, l'amour entre un homme et une femme (à la base de la parenté universelle, tout de même !) doivent-ils être exclus des analyses concernant « les structures élémentaires » de la parenté ? Répondant à ce type d'arguments, ainsi qu'à la critique selon laquelle on ne peut représenter des relations sociales par des symboles formels à la façon des relations mathématiques, Claude Lévi-Strauss rétorqua :

«Qu'entend-on ici par relations sociales ? S’il s'agit de relations concrètes telles que l'observation empirique les appréhende, nous serons d'autant plus facilement d'accord que l'école primaire nous avait déjà enseigné qu'il n'est pas permis d'additionner des poires et des pommes. En revanche, si l'on consent à faire une distinction entre les observations empiriques et les symboles qu'on aura choisi de leur substituer, alors on ne voit pas pourquoi le traitement algébrique de ces symboles - par exemple ceux qui expriment des règles de mariage - ne pourrait pas, à condition qu'on les manipule correctement, beaucoup nous apprendre sur la manière dont fonctionne un système matrimonial, en faisant ressortir des propriétés qui n’étaient pas immédiatement accessibles à l'observation » 41 .

40 Ibid., p. 356.

41 Lévi-Strauss, Anthropologie structurale II, op. cit., p. 99. 
À l'inverse de ce structuralisme a priori, froid et objectiviste du « premier Lévi-Strauss », qui semble cependant rejoindre la réhabilitation de l'objet « institution », code intériorisé mis en avant a posteriori par Radcliffe-Brown, le travail de l'enseignant du Collège de France a fait surgir une problématique plus ouverte. Celle-ci s'est montrée plus attentive à la «fluidité », à la variété, à l'évolution, aux ratés des structures, et, partant, à la subjectivité explicite des acteurs ou à l'historicité organisationnelle, ainsi qu'aux réseaux de relations de ces derniers en situation d'échanges humains concrets, cela, tant dans les sociétés primitives que dans les sociétés historiques que l'ethnologue français s'est attaché à comparer de façon particulièrement stimulante. Sans rechercher des contradictions au sein d’une épistémologie à dominante anglo-saxonne, privilégiant a priori la linguistique structurale et les représentations inconscientes 42 , notons - fait symptomatique - que ce "second Lévi-Strauss », a bien été forcé de réintègrer, in fine, en rejoignant les historiens, les « réseaux » humains concrets...

\section{2. De la structure aux réseaux sociaux concrets et au concept de «maison»}

\section{$\underline{\text { Retour à la table des matières }}$}

À un moment du déploiement de ses définitions fondamentales, la métaphore réticulaire surgit en effet, en filigrane, dans la réflexion lévi-straussienne sur la notion de structure :

« Les systèmes de parenté, les règles de mariage et de filiation, forment un ensemble coordonné dont la fonction est d'assurer la permanence du groupe social, en entrecroisant, à la façon d'un tissu, les relations consanguines et celles fondées sur l'alliance. Ainsi espérons-nous avoir contribué à élucider le fonctionnement de la machine sociale, extrayant perpétuellement les femmes de leurs familles consanguines pour les redistribuer dans autant de groupes domesti-

42 Lévi-Strauss, Anthropologie structurale, op. cit., « Ce que l'ethnographie doit à Durkheim », p. 57-62 ; Le Totémisme aujourd'hui, Paris, PUF, « Mythes et religions », 1962-1971 ; « Introduction à l'œuvre de Marcel Mauss », in Marcel Mauss, Sociologie et anthropologie, Paris, PUF, Quadrige, 1985, p. IX à LII. 
ques, lesquels se transforment à leur tour en familles consanguines, et ainsi de suite (souligné par nous) » 43 .

Un peu plus loin est affirmée l'idée que le structuralisme ne dédaigne pas la morphologie sociale, les systèmes de référence spatiaux et temporels des sociétés primitives. L'apport de l'École de Chicago, attentive à la structure spatiale des villes et aux réseaux sociaux territorialisés, est apprécié ${ }^{44}$. Si l'ethnologie analyse les petits groupes dans un autre contexte, séparément par rapport aux facteurs naturels, elle dépasse l'approche de formes spatiales isolées en les reliant à des systèmes symboliques qui leur donnent sens, car ceux-ci dépendent de propriétés formelles, de configurations mentales liées à des aspects multiples de la vie sociale. Claude Lévi-Strauss valorise au passage la démographie, qui insiste, elle, sur les liens entre le fonctionnement de la structure sociale et l'effectif de la population, de même que sur les propriétés formelles des groupes. Soudain, sans qu'on l'attende, surgit l'objet et le concept de « réseau » :

«Le réseau d’individus défini par les relations d’intermariage est même dans une société moderne - de taille très inférieure à ce qu’on aurait pu supposer à peine dix fois plus grande que celle des plus petites sociétés dites primitives, c'est-à-dire du même ordre de grandeur. Faut-il en conclure que les réseaux d'intermariage sont à peu près constants, en taille absolue, dans toutes les sociétés humaines? Dans l'affirmative, la nature complexe d'une société résulterait moins d'une dilatation de l'isolat primitif, que de l'intégration d'isolats relativement stables dans des ensembles de plus en plus vastes, mais caractérisés par d'autres types de liens sociaux (économiques, politiques, intellectuels) [...].

Tout cela est essentiel, parce que l'ethnologue peut espérer, grâce à ces travaux, retrouver dans une société moderne et complexe des unités plus petites, de même nature que celles qu'il étudie le plus souvent. Néanmoins, la méthode démographique doit être complétée d'un point de vue ethnologique. La taille absolue des isolats n’épuise pas le problème ; on devra aussi déterminer la longueur des cycles matrimoniaux. Toutes proportions gardées, un petit isolat peut consister en un réseau de cycles étendus (du même ordre de grandeur que l'isolat

43 Lévi-Strauss, Anthropologie structurale, op. cit., p. 369.

44 Ibid., p. 346. 
lui-même) ; et un grand isolat peut être fait (un peu à la façon d'une cotte de mailles) de cycles courts. Mais alors, il devient nécessaire de dresser des généalogies, c’est-à-dire que le démographe, même structuraliste, ne saurait se passer de l'ethnologue » 45 .

Dans un souci d'élargissement de l'anthropologie structurale, Claude LéviStrauss va redécouvrir l'objet « réseau » dans l'analyse serrée des mécanismes de dépassement de la parenté, tant au sein des sociétés primitives que dans les «structures diachroniques» des sociétés historiques et urbaines. Au-delà de la parenté, cela revient à prendre en compte les modes de résidence, les « terminologies », les attitudes, ou encore les structures de transmission des règles, celles de dominance (masculine surtout) et de subordination (relevant de l'anthropologie politique).

L'initiateur du « structuralisme » insiste sur le fait que si l'on peut s'interroger sur les liens moins évidents que ne le croyait Radcliffe-Brown entre le « système des attitudes » et le "système terminologique des appellations », on ne peut nier qu'il existe des corrélations entre des positions statiques dans la structure de parenté (réduite à sa terminologie) et les conduites dynamiques des individus, exprimées plus ou moins consciemment dans les statuts, les droits, les devoirs, les obligations, les privilèges, les prohibitions, etc. L'ethnographie a observé que les agents résolvent souvent les contradictions entre les systèmes, adaptent ou font évoluer les règles. La parenté se trouve alors couplée de façon diffuse à d'autres types d' " ordres », comme l'organisation sociale, les stratifications sociales ou économiques (par exemple le système des castes), le politique. Tantôt transitifs et cycliques, tantôt intransitifs et non cycliques, ces ordres sont eux-mêmes ordonnés et hiérarchisés en un "ordre des ordres ». De fait, sur le terrain, tout s'entremêle. Les guerres, les migrations, les intermariages, les échanges avec des sociétés voisines, etc. ont souvent brassé les populations. Les sociétés primitives n'échappent pas à l'histoire! Les systèmes de parenté sont complexes et beaucoup changent sans cesse, perturbés qu'ils sont par des rapports de proximité, par des systèmes d'intégration supérieurs, voire par des systèmes extérieurs qui imposent de nouvelles règles 46 .

45 Ibid., p. 349-350.

46 Unesco, Sens et usages du mot structure, op. cit., p. 42. 
Dépassant les premiers modèles sommaires de Morgan et de Maine, ou ceux de Fortes et d'Evans-Pritchard sur l'Afrique ${ }^{47}$, qui opposaient systématiquement parenté et territoire, dans son enseignement dialectique, Claude Lévi-Strauss a longuement porté son attention comparatiste aux «systèmes bilinéaires » qui déroutent l'observateur de terrain. Ceux-ci peuvent, par exemple, reposer sur des droits fonciers d'attribution ou de partage des terres, non plus sur des règles de filiation. Les individus effectuent alors des choix intentionnels et volontaires (plus que structurels et inconscients), mais limités et changeants selon l'évolution de la structure sociale. Les alliances, les échanges sont, ou directs ou indirects, ou restreints ou généralisés. Les modes d'ascendance ou de descendance peuvent aussi être disjoints et connaître des cycles qui avantagent tantôt les relations matrilinéaires, tantôt les relations patrilinéaires, tantôt indifféremment les unes ou les autres. Ainsi, les relations sociales se compliquent, tant dans les attitudes, les dénominations, que dans leur théorique programmation structurelle. Par exemple, dans sa description de l'orientation patrilatérale des Bororo du Brésil, Claude Lévi-Strauss souligne que chaque mariage créé des lignées inégales et asymétriques qui s’inscrivent « dans un réseau plus général de relations égalitaires et symétriques exprimables seulement au niveau des moitiés » 48 . Ledit système de parenté se trouve alors altéré par des «liens réciproques » entre clans, sous-clans et lignées qui relèvent de moitiés opposées. Les alliances matrimoniales ne constituent qu'une partie des liens en question et se trouvent en contradiction avec la terminologie de la parenté.

La structure sociale se complexifie tout autant avec l'émergence de rapports de dominance, de statut et de pouvoir, c'est-à-dire avec l'apparition du « politique ». La dominance masculine n'influe pas sur les règles de descendance, puisqu'on la trouve dans des sociétés ou matri- ou patrilinéaires. Il en est de même pour le statut des preneurs et des donneurs de femmes. Si le pouvoir des preneurs ne recoupe pas nécessairement leur statut, s'il apparaît supérieur à celui des donneurs, surgissent des règles, soit patrilinéaires, soit matrilinéaires. Le cognatisme

47 M. Fortes, E. E. Evans-Pritchard, Systèmes politiques africains, Paris, PUF, Études ethnographiques, 1964.

48 Lévi-Strauss, Paroles données, op. cit., p. 184-185. 
(filiation indifférenciée par rapport à un ancêtre) ${ }^{49}$ valorise indifféremment les hommes et les femmes (contrairement à l'agnatisme qui privilégie un individu descendant d'un même ancêtre par les mâles). Il représente un « cas intermédiaire » permis par l'équilibre entre les groupes échangistes ou entre les sexes. Mais on trouve aussi des «formules hybrides », mélangeant cognatisme et agnatisme. À ce propos, cela ne concerne pas que des sociétés guerrières cherchant à renforcer l'effectif de leurs combattants en intégrant les cognats, en favorisant les adoptions ou les naturalisations. Claude Lévi-Strauss, qui insiste sur la nécessité d'harmoniser un lignage agnatique et une parentèle cognatique qu'il faut mettre à distance, suggère ainsi les liens entre l'élargissement des réseaux et les processus de politisation, cela à travers une première référence médiéviste :

\begin{abstract}
« Dans des sociétés où la dimension du pouvoir coïncide avec celle de la parenté et de l'alliance, la première peut s'exprimer intégralement ou principalement par l'autre. Au contraire, quand un décollage se produit, le langage de la parenté cesse d'être pertinent, et on glisse vers celui de la résidence (qui a un ou plusieurs chefs) et de la rivalité politique. En ce sens, on a prêté une grande attention à la coexistence, dans plusieurs sociétés de la Nouvelle-Guinée, de ce que les médiévistes européens appellent les "noms de race" et les "noms de terre", et à la manière dont, comme en Europe, les premiers peuvent s'effacer derrière les seconds » 50 .
\end{abstract}

Autre exemple d'ouverture à l'histoire, qui a retenu toute l'attention de l'ethnologue structuraliste sur plusieurs années : le système des «maisons », analysé à partir de cas concrets (dont ceux des Indiens Kwakiutl de la Colombie britannique, des Indiens Yurok de Californie - qui utilisent le terme dans leur propre langue ! - mais aussi de diverses populations en Indonésie, Mélanésie, Polynésie, Nouvelle-Zélande, Madagascar, Micronésie, Afrique). La «maison » est une institution complexe qui dépasse les familles, les clans, les lignées, les habitations. Elle inclut les agnats et cognats, des parents éloignés, des alliés et des clients qui

49 Cf. Lévi-Strauss, Les Structures élémentaires de la parenté, Paris, Mouton, De Gruyter, 1948-2002, et pour une initiation, Christian Ghasarian, Introduction à l'étude de la parenté, op. cit.

50 Lévi-Strauss, Paroles données, op. cit., p. 204. 
possèdent ensemble des biens matériels et immatériels. Comme dans le cas des maisons médiévales européennes, on se trouve en présence d'une « personne morale détentrice d'un domaine, qui se perpétue par transmission de son nom, de sa fortune et de ses titres en ligne réelle ou fictive, tenue pour légitime à la seule condition que cette continuité puisse s'exprimer dans le langage de la parenté ou de l'alliance, et le plus souvent, des deux ensemble » ${ }^{51}$. Claude Lévi-Strauss ajoute :

« En Europe et dans d'autres parties du monde, les maisons médiévales présentent exactement les mêmes caractères. Elles aussi se définissent d'abord par la possession d'un domaine composé de richesses matérielles et immatérielles - les « honneurs - et au nombre desquelles figurent même des trésors d’origine surnaturelle. Pour se perpétuer, les maisons font largement appel à la parenté fictive, qu’il s’agisse de l'alliance ou de l'adoption. À défaut d'héritiers mâles, et parfois concurremment avec eux, les sœurs et les filles peuvent assurer la transmission des titres soit de plein droit, soit en faisant, comme on disait alors "le pont et la planche" ; ainsi que c'est la règle chez les Kwakiutl, elles transmettent alors à leurs enfants des prérogatives que ceux-ci tiennent, par son intermédiaire, de leur grand-père maternel. D’où, peut-être, dans des régimes ostensiblement patrilinéaires, la place importante souvent faite au matronyme.

Enfin, dans toutes les sociétés “à maisons”, on observe des tensions et parfois des conflits entre des principes antagonistes, ou qui sont ailleurs mutuellement exclusives : filiation et résidence, exogamie et endogamie, et, pour employer une terminologie médiévale mais qui s’applique parfaitement aux autres cas, droit de la race et droit de l'élection.

Pour conclure, on s'est interrogé sur les caractères communs de la structure sociale propres à expliquer cette récurrence des mêmes institutions chez des peuples fort éloignés dans le temps et dans l'espace. Il a semblé qu'ils trouvent leur origine dans un état de la structure où les intérêts politiques et économiques tendant à envahir le champ social ne disposent pas encore d'un langage distinct, et, contraints de s'exprimer dans le seul disponible qui est celui de la parenté, doivent inévitablement le subvertir $\gg 52$.

51 Ibid., p. 190.

52 Ibid., p. 190-191. 
Sur ces problèmes, l'ethnologie anglo-saxonne a toujours éprouvé des difficultés à analyser l'objet complexe qu'est « la maison ». Prisonnière de ses catégories sociologiques séparées les unes des autres et aussi de ses conceptions juridiques transposées, elle a eu du mal à relier les rapports de parenté (alliances, filiation), d'ascendance et de descendance, de propriété, de résidence, en l'absence de règles de successions, liées comme au Moyen Âge à un office ou à un domaine héréditaire. Certains observateurs (les Geertz pour Bali, par exemple) ont ainsi parlé de « lignage », de " caste », d' « association culturelle », de « faction » ou de " parti politique »... Même si la maison médiévale européenne comprenait tous ces aspects, il s'agit là de désignations décalées. Loin d'être une institution coutumière locale (les ethnologues anglais, proches de leur droit, utilisent volontiers le terme impropre de corporate groups), ou une personne morale hors de tout statut, la maison apparaît durable (fait pressenti par le droit romain écrit qui parlait, lui, de personae vice fuguntur) ${ }^{53}$. Elle implique l'émergence de réseaux inédits de relations sociales qui relèvent tout autant d'une approche "substantivistes " des groupes réels que d'approches formelles déchiffrant des systèmes de règles dont le contenu inconscient apparaît variable et la durée assez brève. Les «maisons » reposent plus sur l'alliance que sur la descendance, la propriété, la résidence, elles, toujours instables.

Claude Lévi-Strauss s'intéresse alors à ses aspects fonctionnels (mais est-il là si éloigné que cela de la problématique inversée de Radcliffe-Brown ?). Il semble, en tout cas, qu'il a découvert le lien entre dépassement de la parenté et émergence du politique en ce qui concerne la gestion d'une collectivité élargie. En effet, la « maison » est « inventée » (que l'on est loin, là des structures sociales « inconscientes »!) pour unifier des groupements rendus fragiles par l'élargissement des familles. Elle permet d'immobiliser, d'hypostasier, de fétichiser sous forme fantasmatique les relations d'alliance entre preneurs et donneurs de femmes, sources permanente de conflits d'intérêt. La représentation consciente de la maison, le décor, l'espace, l'architecture, la répartition des habitants, tout son symbolisme reflète et structure le système des rapports sociaux. La «maison » a pour fonction de rétablir l'équilibre entre agnats et cognats, sous peine d'étendre à chaque génération les effectifs des non-agnats au détriment des cognats. Il s'agit d'éloigner du noyau agnatique une partie des cognats, en triant la parentèle. Ce qui compte,

53 Ibid., p. 192. 
c'est de comprendre, non les oppositions entre systèmes patrilinéaires et matrilinéaires, ou les liens entre mari et femme et frère et sœur, mais les relations entre « les partenaires d'un réseau d'échanges matrimoniaux » 54 .

Les sociétés unilinéaires ne connaissent de changements que venant de l'extérieur (migrations, guerres, disettes, épidémies, soumissions coloniales...). Elles restent statiques, réglementées. Sans se montrer évolutionniste, Claude LéviStrauss constate qu'à l'inverse, les sociétés cognatiques produisent elles-mêmes leur propre histoire. Le cognatisme donne un «fondement naturel à la culture » ${ }^{55}$. La descendance est comme inversée. Faut-il alors réintroduire la « liberté des acteurs », si chère à « la terminologie de Cambridge » (sic), séparer la descendance (plus naturelle) et la filiation (plus optative) ? Claude Lévi-Strauss résiste encore : les sociétés cognatiques inventent non des règles idéales, mais des mécanismes changeants. On peut parler à leur égard de « stratégies », mais cellesci ne peuvent être ni conscientes, ni individuelles - hypothèse structuraliste oblige ! - :

"Ce sont plutôt des stratégies élaborées et mises en œuvre, non par des individus, mais par des personnes morales assurées d'une durée de vie plus longue que les individus qui les composent. Dans de tels cas (pas plus, d'ailleurs, qu'en aucun autre) il n'y a pas d'un côté la société, de l'autre des individus. La force efficiente appartient à des groupes; et ces groupes poursuivent leurs fins propres en fonction de normes qui ne sont pas celles de la société en général, mais de corps intermédiaires qui, dans la société, s'opposent les uns aux autres et rivalisent entre eux $» 56$.

Ces «personnelles morales» semblent correspondre à la définition de «l'institution » avancée par le Doyen juriste de Toulouse, Maurice Hauriou, en 1925. L'ethnologue structuraliste et symboliste redécouvre ainsi, sans fard, le concept d'institution de Radcliffe-Brown! Ces « corps intermédiaires » seraientils des réseaux d'acteurs privilégiés ? En tout cas, c'est bien autour des «maisons » qu'agissent consciemment les agents concernés. Au niveau du mariage, la « maison » permet de choisir (de façon consciente), entre la race (dans la parentè-

54 Ibid., p. 206.

55 Ibid., p. 222.

56 Ibid., p. 223. 
le) et la terre (dans le voisinage et les alliés). Elle se construit aussi par les funérailles, c'est-à-dire au niveau du droit à la tombe, à la fois sol ancestral et chaîne généalogique. Les défunts qui s’y trouvent réunis ont perdu leur individualité distincte en tant qu'agnats, cognats ou affins. Ils font partie d'un "groupe supérieur » 57 . En naturalisant les nouveaux entrants, les «maisons » permettent de dépasser l'instabilité des sociétés unilinéaires, notamment en raison de la plus grande mobilité des hommes qui font la chasse, la guerre et pratiquent des échanges économiques dans un espace de plus en plus élargi. Cependant, cette institution n'empêche pas les conflits entre preneurs et donneurs de femmes, entre paternels et maternels, entre descendants, entre enfants et petits-enfants, ni entre les maisons concurrentes. Cette structure, très fragile, peut aussi se dissoudre au moment des successions.

Claude Lévi-Strauss, attentif au système mental des oppositions logiques binaires qui produit des modèles structuraux dualistes, met l'accent sur les fonctions objectives et collectives de résolution des pôles d'opposition que l'on trouve dans les sociétés primitives : les «maisons » ont " pour fonction » d'intégrer, sans toujours y parvenir, descendance et résidence, exogamie et endogamie, filiation et alliance, droit paternel et droit maternel, hérédité et élection, antiquité et puissance, compréhension (des vertus de la race) et extension (totalité des biens-fonds de la terre) ${ }^{58}$. En tout cas, nous nous trouvons bien en présence d'une institution plus relationnelle, en termes de réseaux, que d'un code symbolique objectif, abstrait, qui programmerait de façon univoque et inconsciente les individus d'une société élargie !

Claude Lévi-Strauss propose alors une hypothèse très « historienne » et passionnante : un tel dépassement des systèmes de parenté apparaît lié à l'émergence de personnages politiques et économiques autonomisés qui ont un pouvoir volontaire, conscient et organisé sur les membres des sociétés qu’ils dirigent...

Sans utiliser le mot « réseau » (mais notons que certains acteurs désignent entre autre la maison par le concept de "grand filet de pêche ", reliant ainsi la « maison » à l'origine étymologique du mot « réseau »!), en insistant sur le fait que les «maisons » sont déconcertantes par leur «fluidité », l’ethnologue nous

57 Ibid., p. 226.

58 Ibid., p. 239. 
suggère, dans le passage suivant de Paroles données, que ce qui échappe à la parenté désormais, malgré l'usage d'une terminologie parentale, prend la forme de réseaux concrets d'acteurs collectifs :

«À l'école [des historiens], [les ethnologues] apprendraient pourtant qu'au Moyen Âge, des formations plus ou moins durables, et hétéroclites par nature et par origine - communes, associations commerciales ou religieuses, guildes, confréries, etc. - purent, à certains moments, acquérir une indépendance et une autonomie comparables à celles dont jouissaient les fiefs ; qu'une commune ne comprenait parfois qu'une minorité des habitants de la ville ; que le pouvoir y était tantôt exercé par l'assemblée de tous les habitants, tantôt restait aux mains de quelques puissants lignages; que la solidarité familiale fournissait un modèle, même fictif, à des associations telles que les guildes, dont les fonctions furent d'abord religieuses avant de devenir aussi, ou surtout, économiques ; enfin, que la communauté populaire put entrer occasionnellement dans la hiérarchie féodale. En dépit, ou plutôt à cause de leur hétérogénéité, tous ces traits [...] n’ont pu converger vers des types de formations sociales compatibles avec la maison féodale que parce que celle-ci les incluait déjà, avec ses sacra, son arbre généalogique, son esprit de caste, ses intérêts économiques et politiques » 59 .

L’auteur reconnaît le caractère « réel » des réseaux de maisonnées :

« En général, ces formations qui s'unissent autour d’une chose réelle ou qui en prennent le nom transcendent, recoupent ou débordent les groupements familiaux et les lignages. Et même quand leur noyau est d'essence lignagère, elles intègrent sans difficulté des membres supplémentaires recrutés sur la base de l'alliance matrimoniale, de la parenté cognatique, du patronage économique ou du parrainage politique $» 60$.

Il faut rappeler qu'à ce niveau d'analyse, Claude Lévi-Strauss rejoint la tradition de l'ethnographie qui a étudié de façon ouverte les sociétés traditionnelles en termes réticulaires, en décrivant sous forme de sociogrammes arborescents les

59 Ibid., p. 199.

60 Ibid., p. 200. 
réseaux de classes d'âge 61 ou en mettant l'accent sur l'existence d'« associations » séparées de la parenté.

Ainsi, dans son Traité de sociologie primitive ${ }^{62}$, Robert Lowie a souligné de façon pionnière l'importance souvent négligée des "groupes associationnels » dans l'organisation sociale primitive. Il a critiqué, force exemples à l'appui, le «système de Schurtz » (Altersklassen und Männerbünde, Berlin, 1902), selon lequel les femmes, peu sociables, seraient centrées principalement sur les groupes de parenté, alors que les hommes, plus ouverts, monopoliseraient la participation à des associations (les rares associations féminines n'étant que de pâles imitations de ces dernières) ${ }^{63}$. Schurtz pensait qu'une différence de psychologie des deux sexes expliquait la structure sociale. Lowie démontre, cas à l'appui, que les présupposés en question ne se vérifient pas. Il discute ensuite l'hypothèse selon laquelle le type d'association le plus ancien serait la division en classes d'âges, qui oppose enfants, adolescents nubiles et couples mariés. Les «clubs masculins » séparent de façon importante les adolescents et les hommes murs, mais s'autonomisent par rapport aux classes d’âge. Développant une sociabilité multiforme, ils peuvent exiger des «droits d'entrée » (excluant les pauvres), assumer des cérémonies, faire office d'ordre religieux, proposer des festins de bonne camaraderie. Il faut aussi compter, en dehors des systèmes de parenté, les « sociétés secrètes », avec leurs épreuves et cérémonies initiatoires, orientées souvent autour d'un culte des ancêtres, d'un usage des masques, d'une culture mystique des crânes ou de tabous multiformes pour sauvegarder les propriétés, voire instituées pour terroriser les non-initiés ou pour promouvoir des pratiques médicales et incantatoires (les faiseurs de pluie), des cours de justice, comme des espaces de constitution et de reproduction de liens de pouvoir.

Lowie a proposé une typologie de ces associations qui couvrent donc plusieurs fonctions en dépassant les relations de parenté : l'union de deux amis non apparentés (qui se jurent une camaraderie éternelle), forte chez les Indiens Dakota

61 On peut signaler là la réflexion de M. Selz, « Les réseaux de parenté », communication au colloque de Lyon sur la théorie des réseaux et leurs applications en sciences humaines, Université de Lyon I, 1990, poursuivie par la création d'un groupe de recherches sur le thème des réseaux à l'École des Hautes Études de Paris en septembre 2006.

62 Robert Lowie, Traité de sociologie primitive, op. cit.

63 Ibid., p. 279-315. 
par exemple ; les associations shamaniques à la recherche d'une expérience surnaturelle commune (comme chez les Indiens Omaha); les associations de danse (celle des Chiens et des Renards, chez les Indiens Omaha, les Ponka, les Dakota); les associations militaires (chez les Crow ou les Hidatsa), graduées ou non graduées selon l'âge ; les associations à droits d'entrée (en tabac chez les Crow ou dans les associations féminines Cheyenne). Au sein de certaines tribus indiennes des Prairies, dominent les sociétés d'âge (une dizaine), qui ont chacune leur danse, leurs chants, leurs accessoires, leurs privilèges. Conscient de l'importance de ces phénomènes qui séparent les hommes des sociétés primitives de la parenté, attaché à une conception historique rigoureuse de l'apparition des diverses associations, refusant des généralisations hâtives, Lowie nous met en garde contre des théories sociologiques qui prétendraient les expliquer de façon univoque :

« La recherche de lois d'évolution embrassant la totalité des phénomènes, à la manière des théories de Morgan ou de Schurtz, est une entreprise chimérique. Seule une étude ethnographique intensive de chaque zone culturelle peut établir la succession véritable des diverses phases » 64 .

Opposé aux « incohérences atomistes » de Morgan quant à sa théorie de la « démocratie naturelle » des sociétés traditionnelles (l’Afrique ne pullule-t-elle pas de chefferies et de royautés ?), comme à celle, partagée par Maine, de l'absence de structures territoriales au sein de celles-ci, Lowie ajoute que ces réseaux associatifs sont des «agents politiques potentiels », le maintien de liens de parenté n'étant pas incompatible avec l'existence d’un «État politique » :

« En même temps que la famille et le clan, il a existé pendant un nombre de siècles incalculable des associations, telles les clubs masculins, les classes d'âge ou les organisations secrètes, indépendantes de la parenté, évoluant, pour ainsi dire, dans une sphère toute différente de celle du groupe de parenté et capables de revêtir facilement un caractère politique, si elles ne le présentaient pas dès le début » 65 .

64 Ibid., p. 314.

65 Ibid., p. 367. 
Cela, même si, comme en Chine ancienne, l'existence d'associations n'a pas débouché nécessairement sur la création d'une unité politique. Les associations, ou les guildes peuvent même se révéler «centrifuges ». Des pasteurs nomades comme ceux des plaines de l'Asie ont pu aussi construire des États sans avoir connu de développement associationnel. Lowie conclut :

« Les associations sont un facteur important pour la création d'une solidarité politique, mais non le seul ; même là où elles se rencontrent, tout dépend encore du type d'union qu'elles créent » 66.

Ce n'est donc pas un hasard, au regard de la problématique du dépassement de la parenté par les « maisons » et par les réseaux associatifs, si Claude Lévi-Strauss rendit en 1952 un hommage tant appuyé à Robert Lowie ${ }^{67}$. Celui-ci est décrit comme un chercheur passionné par la connaissance des faits, par la critique des fausses synthèses, des systèmes arbitraires, des mauvaises corrélations, qui a beaucoup apporté au structuralisme sans être structuraliste.

Dans cette logique, nous allons voir que l'œuvre lévistraussienne, évoluant d'une théorie objectiviste, structurale, symbolique, vers une ouverture « historique » aux réseaux concrets dans la confrontation des sociétés traditionnelles aux sociétés complexes, a suscité de façon particulièrement heuristique un dialogue fructueux avec d'autres disciplines scientifiques sur ces sujets. La "maison », structure d'élargissement incontestable de la parenté, que l'ethnologue structuraliste a analysée de façon spectrale, constituerait-elle le « chaînon manquant » entre parenté et politique?

66 Ibid., p. 361-368.

67 Cf. Anthropologie structurale, op. cit., p. 367-368. 


\section{Seconde Partie \\ De la parenté au politique}

\section{$\underline{\text { Retour à la table des matières }}$}

Les ouvrages et les cours au Collège de France de Claude Lévi-Strauss, publiés en résumé dans Paroles données, bouteilles jetées à la mer, semblent avoir éclairé certaines recherches consacrées aux sociétés, non plus « sans écriture », mais « historiques ».

Si les «disciplines » officielles n’ont pas toutes su intégrer les apports du structuralisme, comme le souligne Anita Guerreau-Jalabert 68, notamment en raison de divisions bureaucratiques académiques, certains travaux interdisciplinaires ont cherché à démêler, selon leurs objets, les liens entre parenté, parentèle, clientèle, pouvoir et territoire dans la longue durée, cela en s’inspirant amplement des apports de l'anthropologie sociale, voire du concept lévi-straussien de «maison ».

Une telle problématique a ainsi été utilisée pour approfondir le processus de construction du politique dans l'Europe moderne, en relation avec la délicate question de l'élargissement de la parenté - même ci celle-ci n’explique pas tout et du passage «au territoire». Les médiévistes, les premiers, passionnés d' « anthropologie historique », se sont penchés volontiers sur deux formes générales de dépassement social, idéologique et politique de celle-ci, reliées entre elles : l'une, fictive et culturelle, véritable code symbolique forgé par l’Église catholique ; l’autre, « élargie », émanant de la société civile, cherchant à échapper à la première, qui peut être perçue comme ayant favorisé la multiplication de formes politiques inédites, «néo-parentales » en quelque sorte, s’exprimant, comme l’écrit Claude Lévi-Strauss, dans le langage de la parenté, mais la dépassant.

68 Anita Guerreau-Jalabert, « Note critique. Sur les structures de parenté dans l’Europe médiévale », Annales. Histoire, Sciences sociales, 1981, vol. 36, nº 6, p. 1028-1049. 


\section{La transfiguration chrétienne de la parenté réelle}

$\underline{\text { Retour à la table des matières }}$

L’œuvre de Jacques Le Goff, qui s’est souvent référé à Claude Lévi-Strauss, notamment dans l'analyse des mythes médiévaux ${ }^{69}$, a stimulé, cela va sans dire, une période de créativité pour l'anthropologie historique du Moyen Âge. Le théoricien du «long Moyen Âge » s’est d'ailleurs attaché, dans tous ses travaux, à décrire maints « réseaux » concrets de l’époque : techniques et communicationnels (routes, monastères, abbayes...), sociaux et économiques (clercs, laïcs, marchands, ermites...) ou institutionnels. Sur ce dernier point, il a souvent insisté sur

le caractère réticulaire de l’Église romaine elle-même, qui avait à maîtriser une organisation bureaucratique support de sa politique symbolique « de parenté fictive ». Par exemple, Jacques Le Goff décrit ainsi la tentative de contrôle de la chrétienté par la papauté et la curie romaine, notamment lors des conciles œcuméniques :

« Un élément essentiel du pouvoir des administrations est la maîtrise des distances à l'intérieur des aires géographiques sur lesquelles elles s'exercent. Cette maîtrise est celle d'itinéraires, c’est-à-dire l'organisation des déplacements d'hommes et d'informations dans l'espace et le temps. Cette maîtrise est double. Elle est matérielle, comportant un système de courriers, de relais, des réseaux de nouvelles, etc. Mais elle est aussi intellectuelle, se manifestant notamment par une capacité efficace de prévision.

La curie romaine, au XIII ${ }^{\mathrm{e}}$ siècle, est particulièrement intéressée par ce problème. Centre d'impulsion de toute la Chrétienté, obligée d'animer un double mouvement incessant d'accueil des responsables ecclésias-

69 Jacques Le Goff, Pierre Vidal-Naquet, « Lévi-Strauss en Brocéliande. Esquisse pour une analyse d'un roman courtois », repris dans Jacques Le Goff, L’Imaginaire médiéval. Essais, Gallimard, Paris, 1985, p. 151- 187. Cf. LéviStrauss, "Histoire et structure », Paris, Annales. Économies. Sociétés. Civilisations, 1971 ; Jacques Le Goff et Leroy Ladurie Emmanuel, « Mélusine maternelle et défricheuse », Paris, Annales. Économies. Sociétés. Civilisations, 1971. On peut lire également, sur l’influence de Claude Lévi-Strauss en histoire, Jacques Le Goff, L’Imaginaire médiéval, 1985, p. 151-187. 
tiques et d'envoi de chargés de mission et de mots d'ordre, contrainte d’enserrer dans son réseau des réseaux laïcs en voie de développement dans le cadre des administrations monarchiques, défiée par l'hérésie qui circule sur une large aire de la Chrétienté, la curie romaine a des raisons anciennes et nouvelles pour contrôler l'espace de la Chrétienté » 70 .

Plus ou moins dans la continuité des réseaux de routes et de villes de l'Empire romain, cette maîtrise constitua une sorte d'exploit, tant la surface sociale et territoriale était grande dans un monde temporel très lent dans la vitesse de déplacement des hommes et des choses, freiné aussi par la traversée d'espaces plus ou moins imaginaires et effrayants (dont l'océan des forêts).

La question de la parenté médiévale, en tant que telle, a été abordée lors du colloque de référence tenu au Palais Farnèse, à l’École française de Rome, dirigé par Jacques Le Goff et Georges Duby en 1974 71. Anita Guerreau-Jalabert en a dressé un compte rendu en $1981^{72}$, insistant en premier lieu sur les difficultés qu'ont eu les historiens à trouver un cadre d'analyse et une terminologie adaptés en la matière. Selon elle, trop souvent, ceux-ci se sont contentés de termes flous et mal définis (« famille », « lignage »...), sans non plus critiquer leurs sources, toujours construites (notamment les « généalogies » d’époque). Le problème étant de dépasser « l'enlisement dans le concret, écheveau foisonnant et par conséquent extrêmement difficile à débrouiller en l'absence d’instruments analytiques abstraits » 73 . Comme la parenté est « un ensemble de relations sociales formant système », l’histoire médiévale a intérêt, poursuit-elle, à assimiler les travaux fondamentaux de Claude Lévi-Strauss dont elle résume les apports essentiels. Sans se référer à l'analyse lévi-straussienne des «maisons » (non publiée en 1974 et 1981), elle décrit de façon ethnologique les caractéristiques de la parenté médié-

70 Jacques Le Goff, « La perception de l’espace de la Chrétienté par la curie romaine et l'organisation d'un concile œcuménique en 1274 », in L'Imaginaire médiéval, Paris, Gallimard, 1985, p. 76-83.

71 Jacques Le Goff et Georges Duby (dir.), Famille et parenté dans l'Occident médiéval, Rome, École française de Rome, Palais Farnèse, 1977.

72 Anita Guerreau-Jalabert, « Note critique. Sur les structures de parenté dans l’Europe médiévale », article cité.

73 Anita Guerreau-Jalabert, « Note critique. Sur les structures de parenté dans l’Europe médiévale », article cité. 
vale, puis insiste sur l'importance du code symbolique imposé par l'Église catholique, à la source de sa domination sur le système social féodal.

En effet, au niveau du mariage, l'institution imposa, de façon négative, des règles d'exogamie, de prohibition de l'inceste et de définition des degrés de proximité parentale, de même qu'un système de parenté extensive (les "parrains » et les «marraines»), ainsi que des contraintes concernant les liens charnels entre époux laïcs au niveau de la parenté réelle et, doit-on ajouter, de l'image de la femme, comme de l'invention fameuse du purgatoire ${ }^{74}$.

Fut aussi instaurée, au-dessus de la société, une "parenté artificielle », à l'intention des clercs, prise dans des règles plus de consanguinité que d'alliance, créant un vaste "réseau de parenté spirituelle » ${ }^{75}$, fondé sur des relations « paternelles », « maternelles », « fraternelles » et sur des «filiations » entre monastères, abbayes, ordres itinérants, sur le plan spirituel. Cette parenté symbolique fictive, intériorisée, fit des rites d'intronisation du clergé des simulations des rites nuptiaux en instaurant une sorte d' ' inceste rituel » produisant une " filiation » fictive entre le «Père » (Pater Noster) et la " Mère » (Mater Ecclesia), les « frères » et les « sœurs ». Que dire de « l'immaculée conception » de la Vierge Marie, au-delà du système de parenté humain ? Par ailleurs, les clercs se virent interdire tout lien de parenté réelle (célibat forcé). L’invention de cette parenté fictive eut pour cause, bien évidemment, la corruption et la dépravation des mœurs de nombre d'ecclésiastiques, d'abbés, d'évêques, qui, vivant « dans le siècle », tentés par le mariage, riches d'héritiers, dilapidaient progressivement les terres et les biens de l’Église et bafouaient leur propre dogme. L'interdiction du mariage concernant l'ensemble des clercs devait y mettre fin. Quant à l'imposition d'une conception tutélaire du mariage et de la parenté des laïcs, elle visa en partie à détruire les liens de parentèles localisées et plus ou moins irréductibles à toute règle.

L'historienne médiéviste insiste enfin sur le rôle et les fonctions de la parenté ainsi placée sous contrôle dans le système féodal mais lui servant de modèle. Elle propose des hypothèses sur le fonctionnement des alliances (telles que les définit

74 Jacques Le Goff, La Naissance du purgatoire, Paris, Gallimard, Folio histoire, 1981.

75 Anita Guerreau-Jalabert, « Note critique. Sur les structures de parenté dans l’Europe médiévale », article cité, p. 1036. 
Claude Lévi-Strauss, fondées sur l'échange généralisé dissymétrique ${ }^{76}$ ), le rapport entre parenté et patrimoine, l'usage des noms et de la terminologie parentale. Ces analyses, résumant et poursuivant l'apport du colloque international de 1974, ont été reprises récemment et développées en partie par Jérôme Bachet dans sa reconstruction de la « civilisation féodale » sur la longue durée, mais encore mises en perspective comparative par Mireille Corbier et par Anita Guerreau-Jalabert elle-même, dans une étude de la parenté complexe à Rome et dans l'Occident médiéval 77. On peut citer encore les travaux ad hoc de Christiane KlapischZuber sur la Maison (le cas de la casa toscane, très masculine, agnatique et misogyne) et sur l'imaginaire médiéval arboriforme de la parenté. 78

Cette invention lente d’une " parenté renversée », transfigurée, généralisée sur un territoire immense, qui s'immisça au-dessus des anciens systèmes de parenté de la Rome antique et de celles des communautés celtes, franques, germaines, vikings..., constituerait-elle un des fondements de la «modernité » ? En tout cas, l'Église, forte d'un code inédit défini par le droit canon, au-delà des modalités d'imposition de règles prohibitives et de moyens de contrôle (pastoral et inquisitorial), suscita aussi, comme en un "effet pervers ", des réactions des sociétés locales, attachées à la maîtrise de leurs propres pratiques.

L'imposition chrétienne de l'exogamie généralisée et du mariage monogame indestructible, inscrit dans la temporalité terrestre-unique et céleste-éternelle, déclencha comme une stratégie compensatoire de « quasi-parenté élargie » émanant de la société civile. Pour divers analystes, celle-ci allait favoriser l'émergence de formes politiques de parenté dérivée, marquées par le «patronage » et le « clien-

76 L'auteur se réfère là à la seconde partie des Structures élémentaires de la parenté.

77 Jérôme Bachet, La Civilisation féodale. De l'an mil à la colonisation de l'Amérique, Paris, Aubier, col. historique, dirigée par Alain Corbin et JeanClaude Schmitt, 2004 ; Mireille Corbier, "Parenté et pouvoir à Rome », in Rome et l'État moderne européen. Études réunies par Jean-Philippe Genet, Rome, École française de Rome, 2007, p. 173-196 ; Anita Guerreau-Jalabert, « Rome et l'Occident médiéval. Quelques propositions pour une analyse comparée de deux sociétés à système de parenté complexe ", in Rome et l'État moderne européen, op. cit., p. 197-216.

78 Cf. Christiane Klapisch-Zuber, La Maison et le nom : stratégies et rituels dans l'Italie de la Renaissance, Paris, Éditions de l'EHSS, 1990 ; L'Ombre des ancêtres. Essai sur l'imaginaire médiéval de la parenté, Paris, Fayard, 2000. 
télisme », soupçonnées déjà par le vieux Fustel de Coulanges dans son Histoire des institutions de l'ancienne France ${ }^{79}$.

\section{II. "Quasi-parenté élargie " et construction des formes politiques modernes}

$\underline{\text { Retour à la table des matières }}$

Les suggestions de Claude Lévi-Strauss concernant les «maisons », déjà présentes dans les sociétés traditionnelles, sont particulièrement suggestives. Sous la féodalité, mais aussi, dans la période «moderne » (du XV ${ }^{\mathrm{e}}$ au XVIII ${ }^{\mathrm{e}}$ siècle), des phénomènes de parenté élargie surgirent en effet, au-delà du système des « Maisons royales », comme des feux follets dans toute l'Europe, en relation avec le développement économico-démographique et avec le processus de construction de pouvoirs politiques issus des forces vives de structures sociales de plus en plus autonomisées, multiformes et conflictuelles (communautés paysannes, communes urbaines, clans aristocratiques, maisons seigneuriales, maisons royales...), jusqu'à ce que l'État absolutiste, recroquevillé sur lui-même, tente de les contrôler progressivement ${ }^{80}$. Indiquons là quelques recherches.

Anita Guerreau-Jalabert a insisté sur la dimension parentale de la logique féodale (liens d'hommages et de vassalité quasi parentaux, confraternité paternaliste entre le Pape et les rois ou les empereurs, transférés ultérieurement aux Rois des États monarchiques face à leurs « sujets » et leur « Cour »...). Selon elle, les « réseaux d'alliance matrimoniale », dans la période, à dominante homogamique (entre des catégories sociales de niveau hiérarchique identique) ont reproduit la structure pyramidale de la société féodale, tout en encourageant la concentration du pouvoir et les liens aristocratiques à distance. Progressivement, un système de " quasi-parenté » se généralisa, fondé sur «l'élargissement des réseaux

79 On peut relire avec profit, à ce propos, l'ouvrage lumineux, comme tous ses ouvrages, de Numa Denys Fustel de Coulanges, Histoire politique de l'ancienne France, t. 5, "Les origines du système féodal », sans parler de l'incontournable Cité antique.

80 On peut se référer là, sur un tel thème, à l'œuvre de Pierre Legendre dans son ensemble, mais aussi aux ouvrages de Louis Marin (Le Portrait du roi) et de Jean-Marie Apostolidès (Le Roi machine et Le Prince sacrifié). 
d'alliances » et de " parrainage » dans des espaces proportionnés à la puissance des familles de base, paysannes, bourgeoises ou aristocratiques. D'où l'importance de l'usage du langage de la parenté pour définir des objectifs sociaux et politiques de la part des différentes strates de pouvoir, des réseaux sociaux et des communautés.

Jean-René Trochet, en recherchant les fondements anthropologiques de l'origine de l'État-Nation en Europe occidentale, rejoint en partie ces préoccupations lévi-straussiennes et historiennes ${ }^{81}$. Le promoteur de la géographie historique défend l'idée que la parenté réelle est en fait restée cantonnée à un niveau local. À ce sujet, l'anthropologue Pierre Lamaison a insisté sur le fait qu'il existait bien trois modèles de dévolution des biens liés à des systèmes juridiques différents (romain, normand, basque...) : les « systèmes à parentèle » (succession fractionnée, héritage égalitaire, maisonnées individuelles et ménages mobiles), les «systèmes à maison » (succession unique, héritage accordé à l'avantage d'un héritier - préciputaire -, maisons patrimoniales) et les «systèmes de lignage » (succession fractionnée, héritage sélectif, hameaux ou quartiers lignagers), répartis spatialement de façon différenciée dans toute l'Europe ${ }^{82}$. Mais ceux-ci, cantonnés à un niveau domestique et familial, n'ont exercé aucune influence sur l'organisation du territoire. La parenté n’a été impliquée que dans des formes limitées de coopération locales fonctionnelles, sans marquer les formes territoriales officielles. En Occident, poursuit Jean-René Trochet, contrairement à l'Europe orientale où les structures de parenté sont restées dominantes, la construction d'un espace politique dans une société en expansion démographique constante est passée plus par l'organisation d'une territorialité marquée par le droit romain, puis par le « maillage paroissial » chrétien (véritable « réseau territorial »), que par les règles de parenté réelle. Seule l'Irlande, l’Écosse et le Pays de Galle ont conservé

81 Jean-René Trochet, «Parenté et territoire entre l'Antiquité tardive et le haut Moyen Âge en Europe occidentale, et spécifiquement en France », in Le Voyage inachevé. Hommage à Joël Bonnemaison, Paris, Orstom, 1998, p. 193-197 ; «Sur quelques fondements méconnus de l’État-Nation en Europe. L'avènement du réseau paroissial et la promotion de la famille conjugale ", in Géographie et Liberté. Mélanges en hommage à Paul Claval, Paris, L’Harmattan, 1999, p. 283-289.

82 Cf. Pierre Lamaison, Généalogie de l'Europe, Atlas de la civilisation occidentale, Paris, Hachette, France Loisirs, 1995. 
des structures « claniques-lignagères ». Trochet oppose les cultures communautaires, conjugales et domestiques, aux cultures verticales de l'Église, puis de l’État. Il parle de la « lente pénétration du maillage paroissial dans les campagnes » qui effaça la logique de la parenté en imposant un modèle territorial de référence et d'identité, lié à la promotion de l'individu et de la famille conjugale par l'Église.

Le géohistorien insiste à l'inverse, dans une seconde étude sur la disparition des groupes de parenté dans les processus de structuration du territoire en Occident ${ }^{83}$, sur l'importance du modèle romain de territorialisation autonome, qui dépassa une parenté à dominante matrilinéaire, variable selon les lieux et les cités dans la majorité de l'Europe occupée depuis Auguste. Dès l'instauration de ce modèle administratif, les groupes de parenté perdirent leur capacité de dire le droit et d'organiser le territoire. Cela s'aggrava, en raison des conceptions de l'Église, hostile à l'aristocratie, mais aussi de par la prégnance des théories germaniques privilégiant la dominante agnatique et exogamique. Trochet précise que diverses structures de « famille élargie » se substituèrent à celles existantes, alors qu'en même temps perdurait l'organisation romaine avec l'installation des évêchés dans les anciennes civitae. La monarchie franque instaura un système multilinéaire, rendant difficile une mémoire généalogique. Et le géographe de conclure :

«L'uniformisation religieuse par le christianisme et la conception de l'individu qu'il portait, se superposèrent donc à un prolongement du droit romain par le droit canonique, en matière de conception du mariage et de la famille, et au maintien des circonscriptions territoriales de base de l'Empire romain à travers les divisions épiscopales. Cette conjonction entre la promotion de l'individu et du mariage d'une part, que l'Église s'efforça de dégager des liens parentélaires, et la survie des cadres territoriaux romains par l'intermédiaire de l'Église d'autre part, fut l'un des éléments clés de la transition entre l'Antiquité et le Moyen Âge en Europe occidentale. Il faut y voir l'une des origines lointaines des États-nations européens » 84

83 Jean-René Trochet, « Parenté et territoire entre l’Antiquité tardive et le haut Moyen Âge en Europe occidentale, et spécifiquement en France », in Le Voyage inachevé. Hommage à Joël Bonnemaison, op. cité, cf. note 81.

84 Idem, p. 197. 
L’historien Yves Durand, à la suite de Roland Mousnier, mais sans référence à Claude Lévi-Strauss, avait proposé antérieurement une typologie descriptive et comparative des structures sociales, distinguant les sociétés à classes sociales, les sociétés de castes, les sociétés d' " ordres » - ces deux derniers types incarnant des statuts sociaux, des rôles rigides, une symbolique somme toute assez fermée, ségrégative et hiérarchisée ${ }^{85}$. Il a repéré en leur sein des solidarités égalitaires à dominante hiérarchique, analysant les liens réticulaires dans les «maisons » nobles et les lignages, dans les clans puis dans les clientèles, à partir d'exemples pris dans l'Antiquité, au Moyen-Âge, à l'époque moderne ou dans le contexte de la Cour absolutiste, cela, dans le monde rural et urbain. Il a aussi étudié les clans en Écosse et le clientélisme en Corse, comme les «fidélités », au niveau du pouvoir charismatique, du serment, du loyalisme, et montré comment les clans et les lignages ont fonctionné "à la fidélité » personnelle, alors que les clientèles relevaient plutôt de l'intérêt collectivement partagé.

Les recherches de Jacques Heers sur les clans, les réseaux de parentèles et de clientèles dans les cités italiennes illustrent également les liens entre les phénomènes de " parenté élargie » et la construction des partis politiques modernes 86 . En ce qui concerne leur sociabilité intérieure, chaque grande cité marchande fut structurée à partir d'un tissu de réseaux sociaux entrecroisés, appliquant la parenté élargie. On peut repérer la portée politique de cette mise en réseaux des groupes sociaux, dont le fonctionnement contribua à structurer durablement l'univers urbain entre le $\mathrm{XII}^{\mathrm{e}}$ et le $\mathrm{XVI}^{\mathrm{e}}$ siècle. La sociabilité reposa principalement sur des clans familiaux ou supra-familiaux, à forte dimension « tribale » et communautaire. Ceux-ci étaient cimentés par des liens de parenté entraînant des sentiments identitaires forts. Souvent en butte aux souverains royaux, aux institutions patriciennes, à l’Église, ces structures primaires surent se renforcer politiquement et militairement. Certaines accordèrent de l'importance à des relations de consanguinité (les lignages nobles autour d'un ancêtre commun), démultipliés par des adoptions et des fusions de plusieurs groupes. D'autres constituèrent des lignages

85 Yves Durand, Les Solidarités dans les sociétés humaines, Paris, PUF, col. «L'historien », 1987.

86 Jacques Heers, Le Clan familial au Moyen-Âge, Paris, PUF, Quadrige, 1974 ; Les Partis politiques dans l'Occident médiéval, Paris, PUF, col. «L'historien », 1981. 
d’unions et de confréries élargies, reposant sur des relations rituelles de voisinage, d'entraide solidaire, de culte, de liens fonciers, économiques, professionnels, sécuritaires ou politiques, regroupés par le même nom autour d'une symbolique commune (couleurs et cris de ralliement, port des armes et de l'écu, blasons...). Jacques Heers a découvert l'importance de cette structure de noblesse rurale et urbaine socialement différenciée jusque dans un même groupe ${ }^{87}$. Le « clan familial » réticulaire exista dans toute l'Europe, en Toscane, dans l'Italie du Nord, à Venise, au pays basque, dans le Levant espagnol, en Catalogne, dans le midi de la France, mais aussi dans de nombreuses cités du Nord, en Allemagne, en Belgique, dans les Pays-Bas, en Pologne...

Les relations de parentèle élargie et de clientèle réparties en « consorteries » regroupant divers clans («plusieurs rameaux, plusieurs branches ») ont parfois donné naissance à des communes rurales ou à des réseaux sociaux étalés dans l'espace urbain comme dans les propriétés campagnardes et domaniales. Ils intégrèrent des petits bourgeois et des paysans libres, voire des artisans, des boutiquiers, des bâtards, des clients pauvres, des serviteurs, des familiers ou même des esclaves des chefs de familles. Consanguins, vastes (parfois plusieurs centaines d'individus étalés dans un territoire étiré, les familles pouvant avoir quarante bouches à nourrir), exogames, les clans familiaux pouvaient disposer de «bandes de clients » et de «tout un réseau d'alliances » tissé dans le pays d’origine. Ils formèrent aussi des armées ou des milices privées. Ces réseaux distribuèrent faveurs, prêts d'argent, bénéfices, habits aux couleurs du maître ${ }^{88}$. La vie politique des cités médiévales fut en grande partie dépendante du fonctionnement de ces systèmes extra-familiaux d'alliances et de luttes, à la base des « factions » et des premiers partis politiques ${ }^{89}$.

Les clientèles ainsi entretenues favorisèrent le poids des clans, partagés sans cesse entre guerres privées et vendettas familiales. Les divisions se multiplièrent au sein des cités comme dans les quartiers d'une même ville (le cas de la révolte florentine des Ciompi releva par exemple plus d'une vendetta que d'une lutte des

87 Jacques Heers, Le Clan familial au Moyen-Âge, op. cit.

88 Ibid., p. 84.

89 Jacques Heers, Les Partis politiques dans l'Occident médiéval, op. cit. 
classes entre "arts majeurs » et "arts mineurs », si prisée par Machiavel ${ }^{90}$ ). Échappant à toute définition juridique ou institutionnelle (à part l'exception génoise, qui a laissé des traces statutaires), le clan (qui cooptait parfois ses membres) avait des « contours flous ». D'autant qu'il pouvait fusionner avec d'autres entités familiales, non sans querelles ou risques d'éclatement. La concentration, contre-feu à la multiplication des clans, renforça les hautes familles les plus dynamiques. L'isolement précipitait celles en déclin et les contraignait à l'exil, à la disparition ou à la fusion avec des familles dominantes. Les alliances entre clans familiaux constituèrent un enjeu décisif pour la survie économique et génitrice de chacun. L'appartenance au clan impliquait la paix, mais aussi l'engagement de ne pas se lier à un autre clan organisé. La pratique réticulaire constitua un élément de reproduction sociale et de survie.

Ces «lignages » furent à la fois prolongés et concurrencés par des liens d'identité plus territoriale produits par les nouvelles divisions topographiques à l'intérieur des cités comme des colonies en relations au loin avec celles-ci. Des réseaux de proximité se mirent en place, fonctionnels et fort utiles pour la défense urbaine, regroupant des sociétés populaires des portes, puissantes et organisées, parallèles aux sociétés des tours et des maisons-tours de pierre en hauteur protégeant les clans à l’intérieur de chaque quartier.

Les communes, qui s'autonomisèrent progressivement par rapport aux clans familiaux qui les structuraient, en fonction de leur extension démographique et économique au XIV ${ }^{\mathrm{e}}$ siècle, tentèrent de casser l'influence de tels réseaux élargis. Cependant les clans nobles conservèrent leur suprématie. Ils se rendirent maîtres des quartiers urbains dont ils imposèrent l'architecture et colonisèrent les postes de direction municipale. Ils organisèrent aussi des fraterna, regroupant des « frères » d'un même clan dans des relations d'échanges au loin, constituant des réseaux économiques et domestiques d'associations familiales.

Giovanni Levi confirme la continuité des phénomènes d'élargissement de la parenté et de l'entrelacement des réseaux de pouvoir dans un microcosme rural

90 Jacques Heers, Le Clan familial au Moyen-Âge, op. cit., p. 127-128. Sur Florence, « ville réseau », qui « frôla la révolution industrielle » (Fernand Braudel), cf. Michel Bergès, Machiavel, un penseur masqué ?, Paris, Bruxelles, Éditions Complexe, col. « Théorie politique », 2000. 
piémontais du XVII ${ }^{\mathrm{e}}$ siècle ${ }^{91}$. Face aux réseaux familiaux, les nouvelles institutions urbaines se structurèrent elles-mêmes en réseaux de villes ${ }^{92}$, pour résister aux réseaux en gestation, ceux administratifs des grands États centralisateurs. L'équipe pluridisciplinaire dirigée par Pierre Lamaison a par ailleurs montré, en ce qui concerne ces derniers, le poids des grands lignages administratifs, celui des familles nobles dominatrices sur leurs terres, à travers des formes politiques variées (fief, comté, duché, royaume, empires...). La «haute politique » fut ainsi accaparée en France, jusqu'à la Révolution, par des familles héréditaires fondant leur domination sur un racisme de sang et de rang. Celles-ci furent attentives à leur héritage, à leur lignée, à leur succession, à la transmission de leur statut et de leurs privilèges. Elles menèrent la guerre à des niveaux variés d'intérêts pour défendre leurs logiques d'alliances matrimoniales, transposées politiquement et militairement de façon plus ou moins violente, ségrégative et élitiste dans le système des relations internationales qui aboutit au conflit de 1914-1918 93 .

Les «familles » et les «partis » de l'Europe moderne et contemporaine ont perpétué, jusqu'aux plus hauts sommets de l'État d'aujourd'hui, des représentations et des pratiques ambiguës en relation avec la parenté. De même, sur le plan politologique, des continents ou des pays entiers (en Afrique, Asie, MoyenOrient, Amérique du Sud, Inde...), des communautés (mafias, tziganes...) subissent ce type de phénomène, à la fois à l'identique et de façon différenciée. Cela semble relier, de façon «invariante » et peut-être "systémique », parenté réelle, élargie, symbolique ou transfigurée, pouvoir et domination, puissance et sexualité ${ }^{94}$. La politique, loin d'être simplement idéologie, organisation, droit, fonctionne aussi aux relations personnelles, au clientélisme, au patrimonialisme.

91 Giovanni Levi, Le Pouvoir au village. La carrière d'un exorciste dans le Piémont du XVII siècle, Paris, Gallimard, 1989.

92 Cf. à ce propos Laurence Bucholzer-Rémy, Une Ville en ses réseaux : Nüremberg à la fin du Moyen Âge, Paris, Belin, 2006.

93 Cf. Pierre Lamaison, Généalogie de l'Europe, Atlas de la civilisation occidentale, op. cit. Cf. également, Arno Mayer, La Persistance de l’Ancien Régime. L'Europe de 1848 à la Grande Guerre, Paris, Flammarion, col. "Champs ", 1983 et Frédéric Mitterrand, Les Aigles foudroyés. La fin des Romanov, des Habsbourgs et des Hohenzollern, Paris, Robert Laffont, France 2 éditions, 1997.

94 Sur le rapport entre hypertrophie sexuelle, parenté et puissance, cf. Georges Balandier, « Le politique des anthropologues », in Traité de Science politique, 
Alors que nombre d'historiens abordent la question de la « parenté élargie » sans se référer aux travaux de l'ethnologie, le concept de «sociétés à maisons » apparaît là fondamental. Claude Lévi-Strauss l'a cependant quant à lui bien distingué des autres formes de parenté usitées par les historiens, montrant que les « maisons » « ne sont ni des clans, ni des septs, ni des gens, ni des lignages ou lignées, ni exactement des familles » et qu’elles fonctionnent dans des sociétés « où s’esquisse une volonté consciente d'ouverture à un devenir historique » 95.

C'est cette hypothèse qu'a investi, à son tour, le sociologue Pierre Bourdieu dans un article heuristique consacré à la genèse de l’État moderne ${ }^{96}$. Réfléchissant sur le passage de l'État dynastique à l’État bureaucratique, il insiste, un peu comme l'ethnologue Pierre Lamaison, sur l'importance des familles, sur leur politique patrimoniale, leurs liens personnels, comme sur le caractère décisif de la « maison », structure économique et sociale originale aux fortes stratégies de reproduction et de perpétuation. Le sociologue, qui plaide pour l'utilisation des « acquis de l'ethnologie » sur les «sociétés à maison », va plus loin. Selon lui, l’État dynastique « peut en quelque sorte se déduire du modèle de la maison » :

« Pour le roi et sa famille, écrit Bourdieu, l’État s’identifie à la “maison du roi”, entendue comme un patrimoine englobant une maisonnée, c’est-à-dire la famille royale elle-même, qu’il faut gérer en bon "chef de maison” (capmaysouè, comme dit le béarnais). Englobant l'ensemble de la lignée et ses possessions, la maison transcende les individus qui l'incarnent, à commencer par son chef lui-même qui doit savoir sacrifier ses intérêts ou ses sentiments particuliers à la perpétuation de son patrimoine matériel et surtout symbolique (l’honneur de la maison ou le nom de la lignée) » 97.

tome 1, sous la direction de Jean Leca et Madeleine Grawitz, p. 309-334, Paris, PUF, 1985.

95 Cf. Lévi-Strauss, « Maison », article du Dictionnaire de l'ethnologie et de l'anthropologie, sous la direction de Pierre Bonte et alii..., Paris, PUF, col. «Quadrige », 2000, p. 434-436.

96 Cf. Bourdieu Pierre, « De la Maison du Roi à la Raison d’État. Un modèle de la genèse du champ bureaucratique ", in Actes de la Recherche en Sciences sociales, vol. 118, juin 1997.

97 Ibid., p. 56. 
N'est-on pas là en présence, jusqu'au niveau du processus de construction de l’État moderne, de cet « intégron » découvert par Claude Lévi-Strauss, qui semble avoir constitué, tant au niveau de la société civile qu’à celui des différentes formes de pouvoir, la structure de transition et le modèle politique entre un type de société et un autre ? Mais qui a aussi, favorisé, en tant que système de parenté élargie, une base sociale parallèle et plus ou moins différenciée par rapport au « programme » de la « parenté fictive » inventé par l’Église catholique ? Ces deux conceptions concurrentes de la parenté, à la fois symboliques et matérielles, n’ayant pu, historiquement parlant, se concilier... Ce qui a donné le processus collectif que l'on sait...

\section{Fin du texte}

